\title{
Highly specific chimeric DNA-RNA guided genome editing with enhanced CRISPR-Cas12a system
}

Hanseop Kim ${ }^{1,3, \#}$, Wi-jae Lee ${ }^{1,4, \#}$, Chan Hyoung Kim ${ }^{1,11}$, Yeounsun Oh ${ }^{1,6}$, Lee Wha Gwon ${ }^{2,5}$, Hyomin Lee $^{8}$, WooJeung Song ${ }^{8}$, Junho K. Hur ${ }^{9,10}$, Kyung-Seob Lim ${ }^{1}$, Kang Jin Jeong ${ }^{2}$, Ki-Hoan Nam ${ }^{7}$, YoungSuk Won ${ }^{7}$, Youngjeon Lee ${ }^{2}$, Young-Hyun Kim² ${ }^{2}$, Jae-Won Huh ${ }^{2}$, Bong-Hyun Jun ${ }^{4,}$, Dong-Seok Lee, and Seung Hwan Lee ${ }^{2,5,{ }^{*}}$

${ }^{1}$ Futuristic Animal Resource \& Research Center (FARRC), Korea Research Institute of Bioscience and Biotechnology (KRIBB), Cheongju, Korea

${ }^{2}$ National Primate Research Center (NPRC), Korea Research Institute of Bioscience and Biotechnology (KRIBB), Cheongju, 28116, Republic of Korea

${ }^{3}$ School of Life Sciences and Biotechnology, BK21 FOUR KNU Creative BioResearch Group, Kyungpook National University, Daegu, Republic of Korea

${ }^{4}$ Department of Bioscience and Biotechnology, Konkuk University, Seoul 143-701, Korea

${ }^{5}$ Department of Biomolecular Science, KRIBB School of Bioscience, Korea University of Science and Technology, Gajeong-dong, Yuseong-gu, Daejeon, Republic of Korea

${ }^{6}$ Department of Biotechnology, College of Life Sciences and Biotechnology, Korea University, Seoul 02841, Republic of Korea

${ }^{7}$ Laboratory Animal Resource Center, Korea Research Institute of Bioscience and Biotechnology (KRIBB)

${ }^{8}$ Department of Medicine, Major in Medical Genetics, Graduate School, Hanyang University, Seoul 04763 , Republic Korea

${ }^{9}$ Department of Genetics, College of Medicine, Hanyang University, Seoul 04763, Republic of Korea

${ }^{10}$ Graduate School of Biomedical Science and Engineering, Hanyang University, Seoul 04763, Republic of Korea

${ }^{11}$ Department of Biological Sciences, Chungnam National University, Daejeon, South Korea

"The authors wish it to be known that, in their opinion, the first two authors should be regarded as joint First Authors.

* To whom correspondence should be addressed. Email: Ish080390@kribb.re.kr

Correspondence may also be addressed to Dong-Seok Lee, Email: lee1@knu.ac.kr, Bong-Hyun Jun, Email: bjun@konkuk.ac.kr. 


\begin{abstract}
The clustered regularly interspaced short palindromic repeats (CRISPR)-Cas12a system is composed of a Cas12a effector that acts as a deoxyribonucleic acid (DNA)-cleaving endonuclease and a crispr ribonucleic acid (crRNA) that guides the effector to the target DNA. It is considered a key molecule for inducing target-specific gene editing in various living systems. Here, we improved the efficiency and specificity of the CRISPR-Cas12a system through protein and crRNA engineering. In particular, to optimize the CRISPR-Cas12a system at the molecular level, we used a chimeric DNA-RNA guide chemically similar to crRNA to maximize target sequence specificity. Compared to the wild type (wt)Cas12a system, when using enhanced Cas12a system (en-Cas12a), the efficiency and target specificity improved on average by 7.41 and 7.60 times respectively. In our study, when the chimeric DNA-RNA guided en-Cas12a effector was used, the gene editing efficiency and accuracy were simultaneously increased. These findings could contribute to highly accurate genome editing, such as human gene therapy, in the near future.
\end{abstract}

\title{
Introduction
}

The clustered regularly interspaced short palindromic repeats (CRISPR)-Cas system, which is known to be a bacterial defense system, is composed of Cas endonuclease and guide ribonucleic acid (RNA); it is known to operate in various living organisms (1-3). Recently, it has been used as a key tool for in vivo therapeutics because it can be reprogrammed specifically for a target gene. Thus, it is easy to use the CRISPR-Cas system to access genetic diseases $(4,5)$. The field of gene therapy is growing into a large market in which these advanced genome editing tools are frequently employed; thus, it is important to determine whether the CRISPR-Cas system can accurately induce mutations into a target $(6,7)$. The target sequence specificity of CRISPR occurs due to molecular-level interactions resulting from its intrinsic properties (8-10). CRISPR-Cas endonuclease recognizes target deoxyribonucleic acid (DNA) based on the complementary nucleotide sequence contained in the guide RNA. CRISPR-Cas recognizes the protospacer adjacent motif (PAM) sequence in the target gene through the PAM interaction (PI) domain, melts the DNA double helix, and propagates the hybridization of guide RNA and target DNA to form a stable R-loop that induces target DNA cleavage (11-14). It has been reported that the hybridization between the guide RNA and the target DNA, which is formed to aid the CRISPR-Cas system in stably binding to the target DNA, is approximately 20-24 bp; it can have various mismatch tolerances depending on the target sequence (15-19). Accordingly, the possibility of inducing cleavage to off-targets similar to the target sequence has been reported, and efforts have been made to reduce such errors $(9,20-22)$.

Among CRISPR-Cas endonucleases, the CRISPR-Cas12a system, which belongs to Class II and type $\mathrm{V}$, has excellent target specificity. Therefore, it has attracted much attention as an accurate genome editing tool for use as a therapeutic agent for human beings in the future $(14,23-26)$. Unfortunately, the CRISPR-Cas12a system has also been reported to have a tolerance for 
mismatches in the intermediate region (8-9 bp), or in the PAM distal region inside the protospacer, which is necessary for target recognition (16). This off-target cleavage effect appears to be more serious for engineered CRISPR-Cas12a, which has enhanced target recognition and improved gene editing efficiency $(27,28)$. When considering gene therapy for human systems in the future, efficiency and safety will likely be important issues; they must be addressed simultaneously to improve the CRISPR-Cas12a system.

In this study, we devised a technology that dramatically lowers the induction of off-target mutations, while efficiently inducing on-target mutations by effectively recognizing various target nucleotide sequences in human-derived cell lines. When using this enhanced Cas12a system (en-Cas12a) with strong target recognition, the average efficiency of inducing mutations in the target sequence increased (1.7-17.16 fold) when guided by a chimeric DNA-RNA guide, compared to the wild-type Cas12a (wt-Cas12a) system. In addition, the average (0.5-10.6\%) of the mutation induction efficiency of off-target nucleotide sequences was reduced (0.1-3.6\%) by using a chimeric DNA-RNA guide, which increased the target specificity 7.6-fold on average. Using the chimeric DNA-RNA guide-based en-Cas12a system developed in this study, it is possible to induce target-specific, high-efficiency gene editing. Therefore, our proof of concept study could contribute to the fundamental treatment of various incurable human diseases resulting from genetic mutations in the near future.

\section{Results}

\section{Comparison of target DNA cleavage activity of chimeric DNA-RNA-guided engineered en- AsCas12a and wt-AsCas12a}

The CRISPR-Cas12a system uses single-stranded crispr RNA (crRNA) to hybridize target DNA with 20 bases, form a stable R-loop, and induce target DNA cleavage. When the amino acid residues (Lys548, Ser542, and Glu174) interacting near the PAM (TTTN) sequence were changed to positive residues for the interaction between Cas12a and the target DNA (Fig. 1a, left inset), the targetinduced indel ratio (\%) was improved for various genes (28). From these results, we speculate that PAM recognition contributes to the kinetics of the entire Cas12a target recognition and DNA cleavage process, and that it can eventually affect stable R-loop formation through the hybridization of DNA and crRNA. The target specificity (on-target editing/off-target editing) of the Cas12a system has previously been optimized by substituting DNA for the 3 '-end of the crRNA to change the hybridization energy between crRNA and target DNA (Fig. 1a, right inset) (29). Based on this system, here we attempted to maximize the target specificity and genome editing efficiency using en-AsCas12a(Acidaminococcus $\mathrm{sp}$. Cas12a), which has enhanced target recognition. First, to improve target specificity by changing the binding energy of the target DNA-crRNA hybridization region, we gradually substituted the crRNA with DNA; we then confirmed the influence of this substitution on the target DNA cleavage for wtAsCas12a and en-AsCas12a effectors (Fig. 1b). Amplicon cleavage assays were performed on the target nucleotide sequences of both genes (DNMT1 and CCR5 site2) (Supplementary Fig. 1). When DNA was gradually substituted from the 3'-end of the crRNA (recognized by AsCas12a), en-AsCas12a showed improved target recognition compared with wt-AsCas12a; it demonstrated more tolerance to 
the DNA substitution of crRNA (Fig. 1b, Supplementary Fig. 2). As previously reported (29), when 12 or more DNAs are substituted from the $3^{\prime}$-end of Cas12a, the cleavage activity of the DNA amplicon is reduced, and almost no activity is shown in substitutions over 16 nt DNAs. However, en-AsCas12a showed robust cleavage activity after 12 nt DNA substitutions at the 3'-end of crRNA, but showed a decrease in activity by more than half following $16 \mathrm{nt}$ or more DNA substitutions. This indicates that en-AsCas12a is more tolerant to DNA substitution at the 3 '-end of crRNA than wt-AsCas12a, which is advantageous for target DNA cleavage.

\section{Optimization of the genome editing activity of engineered en-AsCas12a based on a chimeric DNA-RNA guide to a target nucleotide sequence on the intracellular genome}

To check whether the engineered en-AsCas12a effector, based on this chimeric DNA-RNA guide, could effectively induce target-specific gene mutations in human cells, various chimeric DNA-RNA guides were used to induce mutations and the efficiency was analyzed in comparison with wtAsCas12a. Comparative analysis of mutation induction efficiencies for the target nucleotide sequences of three genes (DNMT1, IL2A-AS1, and CCR5 site1), revealed that engineered enAsCas12a outperformed wt-AsCas12a in terms of editing efficiency (Fig. 2, Supplementary Fig. 1). In particular, the induction of gene mutations targeting intracellular loci showed a different trend from that of amplicon cleavage (Fig. 1b). Unlike wt-AsCas12a, which exhibited a significantly lower operating efficiency based on chimeric DNA-RNA guides for the target sequence in the genome, engineered en-AsCas12a allowed up to 8 nt DNA substitutions (8DNA) from the 3 '-end of the crRNA while maintaining the editing activity (Fig. 2, Supplementary Fig. 3). Surprisingly, engineered enAsCas12a showed 1.5- to 13.6-fold improvements in mutation induction efficiency compared to wtAsCas12a when the 3'-end of crRNA was substituted with 8 nt DNA to increase target specificity. This effect was universally confirmed in various genes (CCR5 site2, FANCF); on average, a 7.3-fold higher recovery was achieved (Supplementary Figs. 3, 4). Previous studies have reported that when AsCas12a targets the intracellular genome and operates based on a chimeric DNA-RNA guide, it is difficult to induce mutations in the target nucleotide sequence due to many restrictions on the topology of the intracellular genome (29). Accordingly, in this study, we attempted to change the genome topology near the target sequence by using nickase. We compared the resulting changes in the genome editing efficiency of the target sequence for wt-AsCas12a and en-AsCas12a (Supplementary Figs. 1, 3, 4). In the case of wt-AsCas12a, the mutation induction efficiency, which was reduced by the use of a 8 nt DNA substituted chimeric DNA-RNA guide (8DNA), was completely recovered by co-treatment with nickase. In the case of en-AsCas12a, the mutation induction efficiency was maintained at a level similar to that of wt-crRNA using chimeric DNA-RNA (8DNA); it was not significantly affected by nickase (Fig. 2, Supplementary Figs. 3, 4). Therefore, these data indicate that using the en-AsCas12a effector, based on the chimeric DNA-RNA guide (8DNA), enables more effective genome editing than wt-AsCas12a when inducing mutations on the target DNA, without the help of nickase.

Improving target specificity for inducing genetic mutations in the intracellular genome using 


\section{chimeric DNA-RNA guide-based engineered en-AsCas12a}

Next, we compared the chimeric DNA-RNA guide-based engineered en-AsCas12a and wt-AsCas12a effectors regarding their target specificities under optimized conditions ( 3 '-end 8DNA substitution of crRNA) that effectively induced mutations in the target sequence (Fig. 3, Supplementary Fig. 1). In the case of wt-Cas12a-based targeting of the CCR5-site1, the target mutation induction efficiency was greatly reduced by the use of chimeric DNA-RNA (Fig. 3a). Unlike wt-AsCas12a, in which mutation induction efficiency was recovered in a nickase-dependent manner, for en-AsCas12a the target mutation efficiency was maintained in a nickase-independent manner by using chimeric DNA-RNA, in which the 3'-end was substituted with 8 nt DNA (Fig. 3b). The nickase dependency was lowered by 11.25 times (Fig. 3c), and target specificity was increased by 2.79 times (Fig. 3d) using en-AsCas12a. In addition, when the chromosome topology near the target sequence was changed using nickase, the target specificity using chimeric DNA-RNA was further increased by 3.45 -fold, compared to that of wt-AsCas12a (Fig. 3d). We further compared the target specificities of the engineered en-AsCas12a and wt-AsCas12a effectors in the target sequences of two other genes (AAVS1-site1 and DNMT1site2) (Fig. 3e-I). When a chimeric DNA-RNA guide was used for AAVS1-site1, neither en-AsCas12a nor wt-AsCas12a effectors showed nickase dependence, and gene mutations were induced with similar efficiencies to conditions using wt-crRNA (Fig. 3e-h). However, when using the en-AsCas12a effector to target the AAVS1-site1 sequence, the indel ratio (\%) was significantly higher (3.9-fold) than that of wt-Cas12a. However, there were also more unintentional mutations in the off-target sequence (off-target1) (Fig. 3e, f). We confirmed that the number of mutations induced in the off-target1 sequence was dramatically reduced by the use of chimeric DNA-RNA, in which the 3'end was substituted with $8 \mathrm{nt}$ DNA. Therefore, the overall target specificity was increased 3.5 -fold compared to that of wt-AsCas12a when chimeric DNA-RNA (8DNA) guided en-AsCas12a was used (Fig. 3h). The DNMT1-site2 showed the same trend as the AAVS1-site1 locus targeted by the Cas12a system (Fig. $3 \mathrm{i}-\mathrm{I})$. In the case of the en-AsCas12a effector based on the chimeric DNA-RNA guide with $8 \mathrm{nt}$ DNA substitution at the 3 '-end, the indel ratio (\%) was significantly increased (1.9-fold) compared to that of wt-AsCas12a. Furthermore, the off-target nucleotide sequence (off-target1, 2)-induced mutations were dramatically reduced (Fig. $\mathbf{3 i}$, j). As a result, the target specificity was increased twofold, regardless of the use of nickase (Fig. 3k, I). In conclusion, by inducing mutations in three genes using chimeric DNA-RNA guided engineered en-AsCas12a, the targeted indel ratio (\%) was improved 7.3fold on average, without the help of nickase. The off-target mutation induction efficiency was also reduced. Eventually, the target specificity was improved 3.1-fold compared to that of wt-AsCas12a.

\section{A model for improving target specificity and mutation induction efficiency of en-Cas12a, based on chimeric DNA-RNA guides}

Combining all of the above findings, the results of the working mechanism of en-Cas $12 \mathrm{a}$, compared to the existing wt-Cas12a, are presented in Fig. 4, based on the chimeric DNA-RNA guide. When wtCas12a was used to cleave the target sequence in the genome of the cell, there was tolerance for mismatches between the protospacer (20bp) middle part and the PAM (TTTN) distal region, so there 
was a possibility that Cas12a could recognize and cleave off-target sequences. When wt-Cas12a, based on the chimeric DNA-RNA guide, was used (in which the 3'-end was substituted with 8 DNAs), the off-target effect could be reduced by changing the binding energies of the target DNA and crRNA. However, when chimeric DNA-RNA based cleavage was performed on the genome in the cell, the mutation-inducing effect on the target nucleotide sequence was largely decreased. So, the target specificity was increased only by changing the topology of the genome with the simultaneous use of nickase.

In the case of en-Cas12a, in which target sequence recognition is reinforced by engineering the interacting amino acids of the PAM sequence recognition part, its efficiency in inducing target sequence mutations for various genes was increased compared to that of Cas12a, but its unintended off-target effects also increased greatly. Using the chimeric DNA-RNA-based en-Cas12a effector to target the intracellular genome can dramatically reduce the effects of off-target mutations. Without the help of nickase, it is thus possible to increase the target sequence editing efficiency and dramatically lower the off-target sequence editing efficiency. Regarding the efficient induction of gene mutations with respect to the use of chimeric guides, accurate and high-efficiency gene targeting is possible when using chimeric DNA-RNA-based en-Cas12a.

\section{Discussion}

The CRISPR-Cas12a effector is attracting attention as a potential future target-specific genome editing tool as it is known to be capable of inducing mutations in a target sequence on a desired gene; it also has the highest target specificity among previously known CRISPR systems. However, the Cas12a system has been reported to have lower activity than Cas9 in general, and there remains room to improve the properties of the endonuclease itself for applications in various in vivo conditions. Efforts have been made to more effectively recognize the target DNA sequence and induce cleavage by engineering the Cas 12 a system $(27,28)$. These studies have shown overall improved activity compared to wt-Cas12a in various genes, through enhanced binding, by changing amino acids around the domain, within Cas12a, and by recognizing the PAM sequence in the target DNA. However, most CRISPR endonucleases induce double-stranded cleavage by forming an R-loop, through the complementary binding of target-strand DNA and crRNA. In general, as the tolerance increases due to enhanced binding affinity, off-target binding also increases. Therefore, we believe that the effects of off-target binding would be maximized when using engineered Cas12a systems, so a method for increasing target specificity is also required, in parallel with methods to enhance activity.

Previous studies have improved target specificity by applying crRNA engineering to the Cas $12 \mathrm{a}$ system; target-specific gene mutations have been effectively induced by optimizing the length of DNA substitution. Mismatch tolerance has been reduced by changing the complementary binding energy of target-strand DNA and crRNA through sequential 8nt DNA substitutions at the 3'-end of crRNA. This principle confirms that the induction of off-target mutations can be reduced while maintaining the efficiency of inducing target mutations. Based on this, here we used chimeric DNA-RNA crRNA to engineer en-AsCas12a, which displayed maximized target sequence recognition and improved 
mutation induction efficiency in various target nucleotide sequences, compared to wt-AsCas12a. Surprisingly, the induction of off-target mutations was dramatically decreased, and eventually the target specificity was largely improved. Interestingly, the observed discrepancy between amplicon cleavage (Fig. 1b) and genome editing inside the cell (Fig. 2) showed that the cleavage activity of Cas12a endonuclease is greatly influenced by DNA topology. Chimeric DNA-RNA crRNA-based enAsCas12a and wt-AsCas12a displayed differing sensitivities to the intracellular genome, so enAsCas12a showed a higher gene mutation induction efficiency than wt-AsCas12a. These results suggest that en-AsCas12a can, to some extent, overcome structures that are unfavorable to target DNA cleavage due to unstable R-loop formation (due to the DNA substitution of crRNA in the genomic sequence). This is achievable by enhancing PAM recognition through protein engineering. In general, the low operating efficiency induced in the intracellular genome by wt-Cas12a (based on the DNA substitution of crRNA to improve target specificity) could be recovered by changing the genome topology by using nickase around the target sequence. However, in the case of en-Cas12a, it was possible to induce original levels of mutation following up to eight DNA substitutions at the 3 ' end of the crRNA, without the help of nickase. Therefore, when using chimeric DNA-RNA guide-based enAsCas12a, it was possible to simultaneously improve the genome editing efficiency (\%) and the target specificity (on-target editing [\%]/off-target editing [\%]) compared to those of wt-AsCas12a by changing the hybridization energy of the target DNA strand and crRNA.

In this study, we developed a technology that maximizes the safety and efficiency of genome editing using chimeric DNA-RNA crRNA-based en-Cas12a. In the future, many improvements are needed in terms of efficiency and safety regarding the application of gene therapy to humans using the CRISPR system, or for sophisticated gene editing in in vivo systems. Through this technology, it is expected that the safety and efficacy of various CRISPR endonucleases can be optimized in a similar way when applied in vivo.

\section{Methods}

\section{Preparation of the CRISPR-Cas12a recombinant protein and chimeric guides}

wt- and en-AsCas12a recombinant proteins were prepared for the in vitro DNA cleavage assay. Codon-optimized AsCas12a (Acidaminococcus sp. Cas12a) coding sequence was cloned into a pET28a bacterial expression vector and then transformed into BL21 (DE3) Escherichia coli cells. Transformed bacterial colonies were cultured at $37^{\circ} \mathrm{C}$ until the optical density reached 0.6 , after which isopropylthio- $\beta$-galactoside (IPTG) inoculation was performed. After $48 \mathrm{~h}$, E. coli cells were precipitated at $4{ }^{\circ} \mathrm{C}$ and $5,000 \mathrm{rpm}$, following which the culture medium of the upper layer was removed. The precipitated $E$. coli cell pellet was resuspended in lysis buffer $[10 \mathrm{mM} \beta$ mercaptoethanol, $300 \mathrm{mM} \mathrm{NaCl}, 20 \mathrm{mM}$ Tris-HCl (pH8.0), $1 \mathrm{mM} \mathrm{PMSF}$, and 1\% TritonX-100]. In order to disturb the bacterial cell membrane, sonication was performed on ice water for $3 \mathrm{~min}$, following which the cell lysate was precipitated for $10 \mathrm{~min}$ at $5,000 \mathrm{rpm}$ at $4^{\circ} \mathrm{C}$. Next, the nitrilotriacetic acid ( $\mathrm{Ni}$ NTA) resin was pre-washed with wash buffer [20mM Tris- $\mathrm{HCl}(\mathrm{pH} 8.0), 300 \mathrm{nM} \mathrm{NaCl}]$ and the precipitated cell lysate was stirred at $4{ }^{\circ} \mathrm{C}$ for 90 min. Washing was performed with ten times the 
volume of wash buffer to remove non-specific binding components in the mixed cell lysate solution. For the elution of AsCas12a protein, an elution buffer $[20 \mathrm{mM} \mathrm{Tris-HCl}(\mathrm{pH} 8.0), 300 \mathrm{nM} \mathrm{NaCl}, 200$ $\mathrm{mM}$ imidazole] was used and finally exchanged against the storage buffer [200 mM NaCl, $50 \mathrm{mM} \mathrm{4-}$ (2-hydroxyethyl)-1-piperazineethanesulfonic acid (HEPES; pH 7.5), $1 \mathrm{mM}$ dithiothreitol (DTT), 40\% glycerol] using a Centricon (Millipore, Amicon ${ }^{\circledR}$ Ultra-15), and stored at $-80{ }^{\circ} \mathrm{C}$. Chimeric DNA-RNA oligonucleotides (Bioneer) were synthesized for each target gene sequence and dissolved in diethyl pyrocarbonate (DEPC) water and then stored at $-80^{\circ} \mathrm{C}$ (Supplementary Table S1).

\section{Preparation of the guide RNA for Cas12a and nCas9(D10A)}

Guide RNAs for Cas12a and nCas9(D10A) were generated by in vitro transcription. A DNA template for in vitro transcription was constructed using annealing or extension polymerase chain reaction (PCR) with sense and antisense DNA oligonucleotides (Macrogen) containing the target DNA sequence (Supplementary Table S2). DNA templates were mixed with T7 RNA polymerase (NEB, M0251L) and reaction buffer mixture $(50 \mathrm{mM} \mathrm{MgCl} 2,100 \mathrm{mM}$ ribonucleoside tri-phosphate (rNTP; Jena Bio, NU-1014), 10X RNA polymerase reaction buffer, $100 \mathrm{mM}$ DTT, RNase inhibitor Murine, DEPC), and incubated at $37^{\circ} \mathrm{C}$. After $16 \mathrm{~h}$, to remove the original DNA template, DNase I was added and the mixture was incubated for another $1 \mathrm{~h}$ at $37^{\circ} \mathrm{C}$. The transcribed RNA was purified using a column (MP Biomedicals, GENECLEAN® Turbo Kit). The purified RNA was concentrated through lyophilization $(2,000 \mathrm{rpm})$ at $-55^{\circ} \mathrm{C}$ for $1 \mathrm{~h}$.

\section{In vitro DNA cleavage assay}

On-/off-target site PCR amplicons of each gene (DNMT1, CCR5, IL2A-AS1, and AAVS1) were obtained from purified human genomic DNA using DNA primers (Supplementary Table S2). The purified target PCR amplicon was incubated with purified recombinant wt- or en-Cas12a protein and crRNA (RNA-guides or chimeric DNA-RNA guides) in 10X buffer (NEBuffer3.1, NEB) for $1 \mathrm{~h}$. After adding a stop buffer [100 mM ethylenediaminetetraacetic acid (EDTA), 1.2\% sodium dodecyl sulfate (SDS)] to stop the reaction, the cleaved fragment was separated using $2 \%$ agarose gel electrophoresis. DNA cleavage efficiency (cleaved fragment intensity [\%]/total fragment intensity [\%]) was measured using ImageJ software $(\mathrm{NIH})$.

\section{Cell culture and transfection}

The HEK293FT (ATCC) cell line was cultured in Dulbecco's modified Eagle medium (DMEM, Gibco) with $10 \%$ fetal bovine serum (FBS, Gibco) at $37{ }^{\circ} \mathrm{C}$ and in $5 \% \mathrm{CO}_{2}$. To ensure efficient chimeric DNARNA guide delivery, we performed sequential transfection with wt- or en-Cas12a expression vectors and crRNAs. For the primary transfection, $10^{5}$ cells were mixed with a plasmid vector (AsCas12a, nSpCas9 (D10A, H840A)) and $20 \mu$ of electroporation buffer (Lonza, V4XC-2032) and were nucleofected according to the manufacturer's instructions (program: $\mathrm{CM}-137$ ). The transfected cells were transferred to a 24-well plate with $500 \mu \mathrm{l}$ of media and incubated at $37{ }^{\circ} \mathrm{C}$ in $5 \% \mathrm{CO}_{2}$. Twenty four hours after primary transfection, for secondary transfection, crRNA (200 pmol), single guide RNA

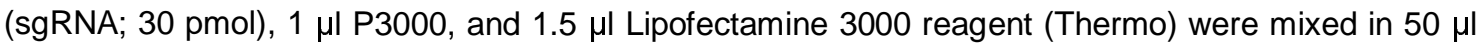


Opti-MEM (Gibco), incubated for $10 \mathrm{~min}$, and added to DMEM media. Forty-eight hours after the second transfection, cells were harvested and genomic DNA was extracted using a genomic DNA purification kit (Qiagen, DNeasy Blood \& Tissue Kit).

\section{Deep sequencing and data analysis}

To analyze the indel frequency of the on-/off-target locus of each gene, targeted deep sequencing was performed using PCR amplicons. The Cas-OFFinder (http://www.rgenome.net/cas-offinder/) web tool was used to select potential off-target sites corresponding to each on-target site. For the preparation of PCR amplicons, PCR amplification was performed using DNA primers corresponding to each endogenous locus (Supplementary Table S2). To add adapter and index sequences to each $5^{\prime}$ and 3 ' ends, nested PCR was performed using Phusion ${ }^{\mathrm{TM}}$ High-Fidelity DNA Polymerase (Thermo). After index tagging, the PCR amplicon mixture was analyzed using a Mini-Seq (Illumina, SY-420-1001) according to the manufacturer's guidelines. Sequencing read fatstq files were analyzed using CasAnalyzer (http://www.rgenome.net/cas-analyzer/), and the indel ratio (mutant DNA read number/total DNA read number) was calculated.

\section{AVAILABILITY}

CRISPR RGEN Tools is an open-source collaborative initiative available in the repository (http://www.rgenome.net/).

\section{ACCESSION NUMBERS}

Targeted deep sequencing data are available at the NCBI Sequence Read Archive (SRA) under the accession number SRP334002.

\section{SUPPLEMENTARY DATA}

Supplementary Data are available at online.

\section{ACKNOWLEDGEMENT}

The authors thank the members of the National Primate Research Center (NPRC) for their helpful discussions.

\section{FUNDING}

This research was supported by grants from the National Research Foundation (NRF) funded by the 
Korean Ministry of Education, Science and Technology (NRF-2019R1C1C1006603) and the Korean Ministry of Science and ICT program (NRF-2017M3A9D5A01072797) through the National Research Foundation and the Korea Medical Device Development Fund grant funded by the Korean government (the Ministry of Science and ICT, Ministry of Trade, Industry and Energy, Ministry of Health \& Welfare, Ministry of Food and Drug Safety) (Project Number: 9991006929, KMDF_PR_20200901_0264). The study was also supported by grants from the Korea Research Institute of Bioscience and Biotechnology (KRIBB; Research Initiative Program KGM5282113, KGM4562121, KGM5382113).

\section{CONFLICT OF INTEREST}

The authors declare that they have no competing interests.

\section{References}

1. R. Barrangou et al., CRISPR Provides Acquired Resistance Against Viruses in Prokaryotes. Science 315, 1709-1712 (2007).

2. M. Jinek et al., A Programmable Dual-RNA-Guided DNA Endonuclease in Adaptive Bacterial Immunity. Science 337, 816-821 (2012).

3. J. A. Doudna, E. Charpentier, Genome editing. The new frontier of genome engineering with CRISPR-Cas9. Science 346, 1258096 (2014).

4. H. Frangoul et al., CRISPR-Cas9 Gene Editing for Sickle Cell Disease and beta-Thalassemia. The New England journal of medicine 384, 252-260 (2021).

5. J. D. Gillmore et al., CRISPR-Cas9 In Vivo Gene Editing for Transthyretin Amyloidosis. 385, 493-502 (2021).

6. X. H. Zhang, L. Y. Tee, X. G. Wang, Q. S. Huang, S. H. Yang, Off-target Effects in CRISPR/Cas9-mediated Genome Engineering. Molecular therapy. Nucleic acids 4, e264 (2015).

7. H. A. Han, J. K. S. Pang, B. S. Soh, Mitigating off-target effects in CRISPR/Cas9-mediated in vivo gene editing. J Mol Med (Berl) 98, 615-632 (2020).

8. S. H. Lee, Y. H. Park, Y. B. Jin, S. U. Kim, J. K. Hur, CRISPR Diagnosis and Therapeutics with Single Base Pair Precision. Trends Mol Med 26, 337-350 (2020).

9. S. Q. Tsai, J. K. Joung, Defining and improving the genome-wide specificities of CRISPRCas9 nucleases. Nature reviews. Genetics 17, 300-312 (2016).

10. J. C. Cofsky et al., CRISPR-Cas12a exploits R-loop asymmetry to form double-strand breaks. Elife 9, (2020).

11. F. Jiang et al., Structures of a CRISPR-Cas9 R-loop complex primed for DNA cleavage. Science 351, 867-871 (2016).

12. D. C. Swarts, J. van der Oost, M. Jinek, Structural Basis for Guide RNA Processing and Seed-Dependent DNA Targeting by CRISPR-Cas12a. Mol Cel/66, 221-233 e224 (2017). 
13. V. Globyte, S. H. Lee, T. Bae, J. S. Kim, C. Joo, CRISPR/Cas9 searches for a protospacer adjacent motif by lateral diffusion. The EMBO journa/38, (2019).

14. S. Stella, P. Alcon, G. Montoya, Structure of the Cpf1 endonuclease R-loop complex after target DNA cleavage. Nature 546, 559-563 (2017).

15. Y. Fu et al., High-frequency off-target mutagenesis induced by CRISPR-Cas nucleases in human cells. Nat Biotechno/31, 822-826 (2013).

16. B. P. Kleinstiver et al., Genome-wide specificities of CRISPR-Cas Cpf1 nucleases in human cells. Nat Biotechno/34, 869-874 (2016).

17. V. Pattanayak et al., High-throughput profiling of off-target DNA cleavage reveals RNAprogrammed Cas9 nuclease specificity. Nat Biotechno/31, 839-843 (2013).

18. S. Q. Tsai et al., GUIDE-seq enables genome-wide profiling of off-target cleavage by CRISPR-Cas nucleases. Nat Biotechno/33, 187-197 (2015).

19. S. W. Cho et al., Analysis of off-target effects of CRISPR/Cas-derived RNA-guided endonucleases and nickases. Genome research 24, 132-141 (2014).

20. J. L. Schmid-Burgk et al., Highly Parallel Profiling of Cas9 Variant Specificity. Mol Cell, (2020).

21. Y. Fu, J. D. Sander, D. Reyon, V. M. Cascio, J. K. Joung, Improving CRISPR-Cas nuclease specificity using truncated guide RNAs. Nature Biotechnology 32, 279 (2014).

22. D. D. Kocak et al., Increasing the specificity of CRISPR systems with engineered RNA secondary structures. Nat Biotechno/37, 657-666 (2019).

23. D. Dong et al., The crystal structure of Cpf1 in complex with CRISPR RNA. Nature 532, 522526 (2016).

24. T. Yamano et al., Crystal Structure of Cpf1 in Complex with Guide RNA and Target DNA. Cell 165, 949-962 (2016).

25. B. Zetsche et al., Cpf1 Is a Single RNA-Guided Endonuclease of a Class 2 CRISPR-Cas System. Cell 163, 759-771 (2015).

26. F. Safari, K. Zare, M. Negahdaripour, M. Barekati-Mowahed, Y. Ghasemi, CRISPR Cpf1 proteins: structure, function and implications for genome editing. Cell Biosci 9, 36 (2019).

27. L. Zhang et al., AsCas12a ultra nuclease facilitates the rapid generation of therapeutic cell medicines. Nat Commun 12, 3908 (2021).

28. B. P. Kleinstiver et al., Engineered CRISPR-Cas12a variants with increased activities and improved targeting ranges for gene, epigenetic and base editing. Nat Biotechno/37, 276-282 (2019).

29. H. Kim et al., Enhancement of target specificity of CRISPR-Cas12a by using a chimeric DNARNA guide. Nucleic acids research 48, 8601-8616 (2020). 


\section{Figure legends}

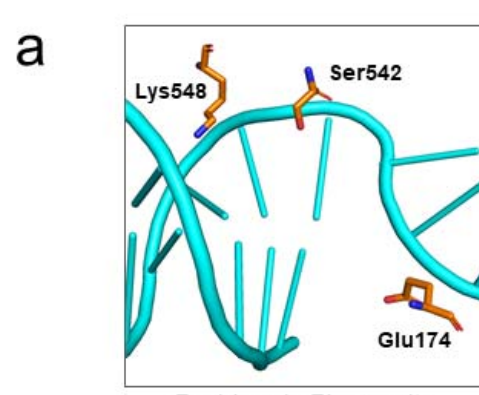

Residues in PI near site

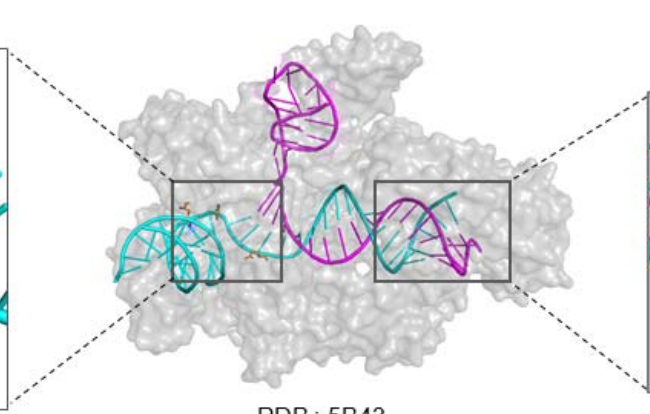

PDB : $5 B 43$ b

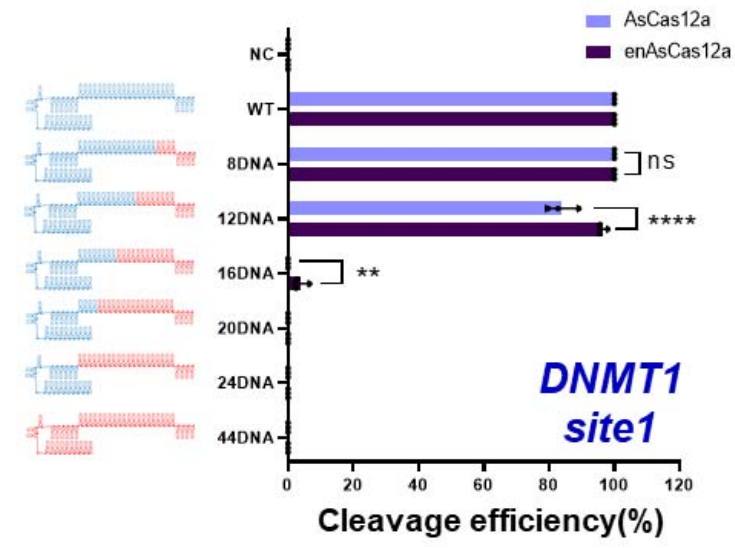

2'-OH contact residues

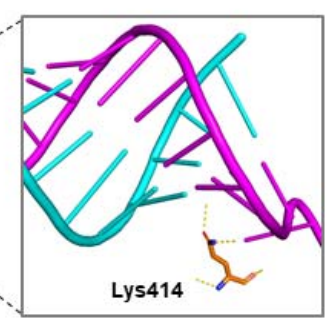

Lys414

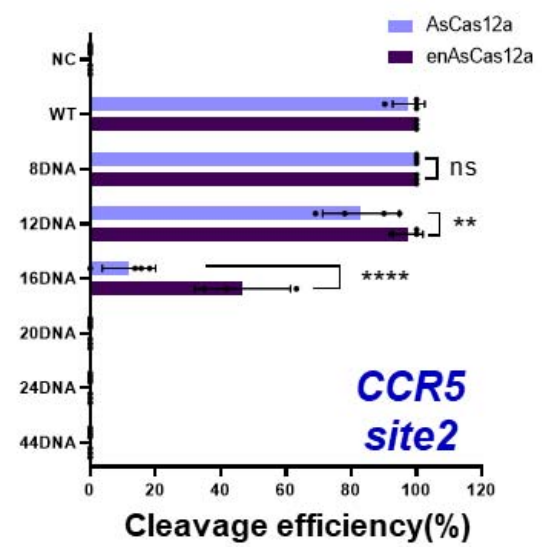

Figure 1. Comparison of target DNA cleavage activity of chimeric DNA-RNA guided en-Cas12a and wt-Cas12a. a, Structure of target-strand DNA-crRNA-AsCas12a complex(PDB: 5B43). left inset: amino acids in AsCas12a interacting with around the PAM sequence in the target DNA, right inset: Amino acid (Lys414) interacting with the target-strand DNA-crRNA duplex in AsCas12a (hydrogen bonding with the 2'-OH group on the crRNA 3'-end side). b, Comparison of cleavage efficiency of wtAsCas12a and en-AsCas12a using a chimeric DNA-RNA guide. Comparison of cleavage efficiency of DNA amplicons including target nucleotide sequences (DNMT1, CCR5-site2) with gradual DNA substitution from the 3 '-end of crRNA. NC: Negative control, WT: Wild-type crRNA was treated with wt- or en-AsCas12a. The RNA region of the (cr)RNA is shown in blue, and the substituted DNA region is shown in red ('8-44DNA' indicates a number of substituted DNA nucleotides in (cr)RNA). The X-axis indicates the efficiency of the target gene (DNMT1, CCR5) cleavage by wt- and en-AsCas12a using various chimeric DNA-RNA guides (DNA substitution from the 3 '-end of the (cr)RNA). All cleavage efficiency were calculated from agarose gel separated band intensity (cleaved fragment intensity (\%) / total fragment intensity (\%)) and normalized to wild-type (cr)RNA (Supplementary Fig. 2). Data are shown as means \pm s.e.m. from three independent experiments. $P$-values are calculated using a twoway ANOVA with sidak's multiple comparisons test (ns: not significant, $\mathrm{P}^{*}:<0.0332, \mathrm{P}^{* *}:<0.0021$, $\left.P^{* \star *}:<0.0002, P^{\star * * *}:<0.0001\right)$. 

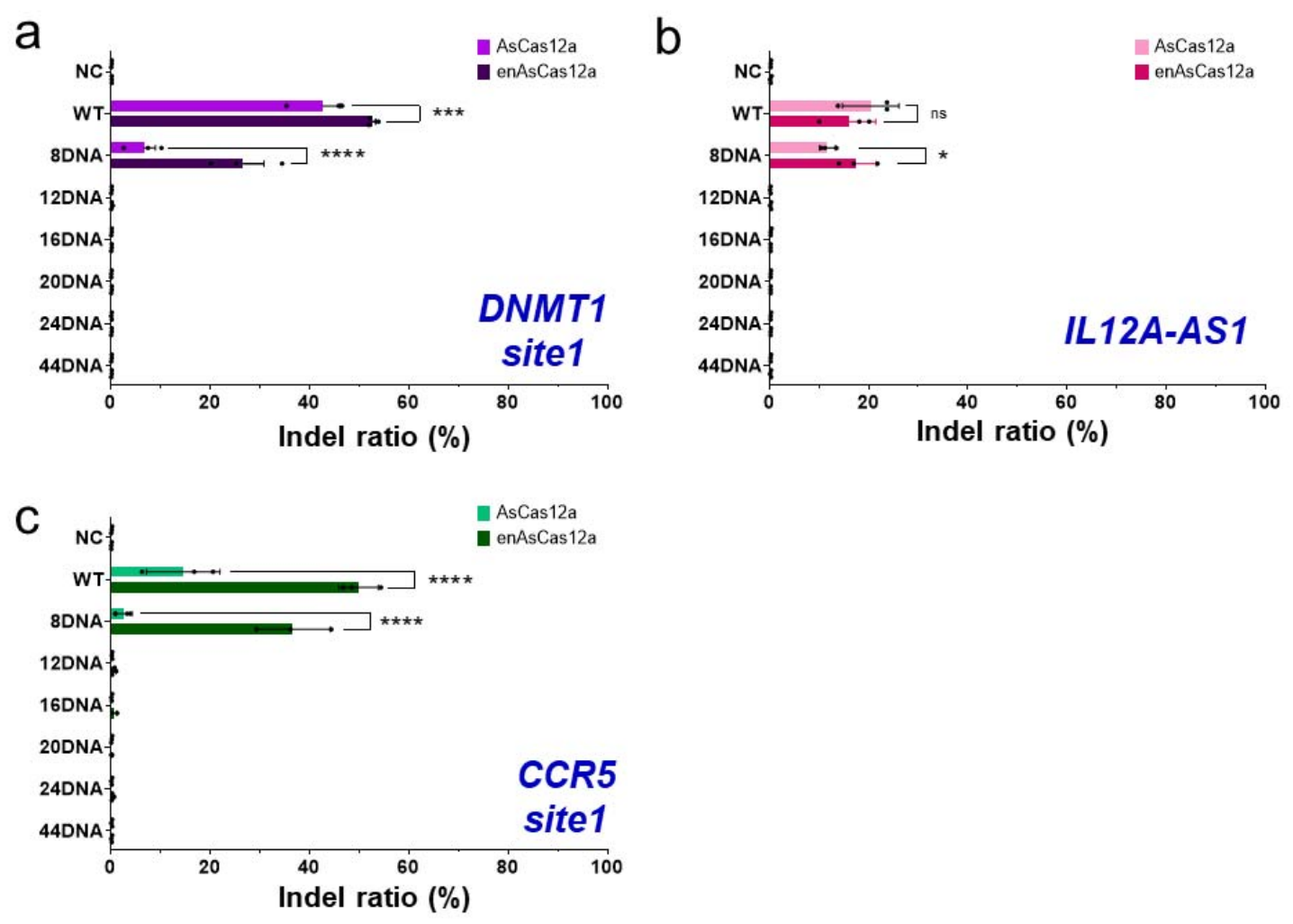

Figure 2. Comparison and optimization of genome editing efficiency of en-Cas12a and wtCas12a based on chimeric DNA-RNA guide targeting intracellular genome. a-c. Comparison of genome editing efficiency (\%) of wt-AsCas12a and en-AsCas12a using a chimeric DNA-RNA guide for human-derived cell line (HEK293FT). Comparison of indel induction efficiency (\%) in intracellular genome target sequences (DNMT1, IL2A-AS1, CCR5-site1) by gradual DNA substitution of the 3'-end of crRNA. All the indel ratio was calculated from targeted amplicon sequencing (Indel ratio(\%) = mutant DNA read number / total DNA read number). Data are shown as means \pm s.e.m. from three independent experiments. $P$-values are calculated using a two-way ANOVA with sidak's multiple comparisons test (ns: not significant, $P^{*}:<0.0332, P^{* *}:<0.0021, P^{* * *}:<0.0002, P^{* * * *}:<0.0001$ ). NC: negative control, WT: Wild-type crRNA was treated with wt- or en-AsCas12a, 8-44DNA: Chimeric crRNA (sequential 8-44DNA substitution at 3'-end of crRNA) was treated with wt- or en-AsCas12a. 
bioRxiv preprint doi: https://doi.org/10.1101/2021.09.04.458978: this version posted September 5, 2021. The copyright holder for this preprint (which was not certified by peer review) is the author/funder, who has granted bioRxiv a license to display the preprint in perpetuity. It is made available under aCC-BY-NC-ND 4.0 International license.

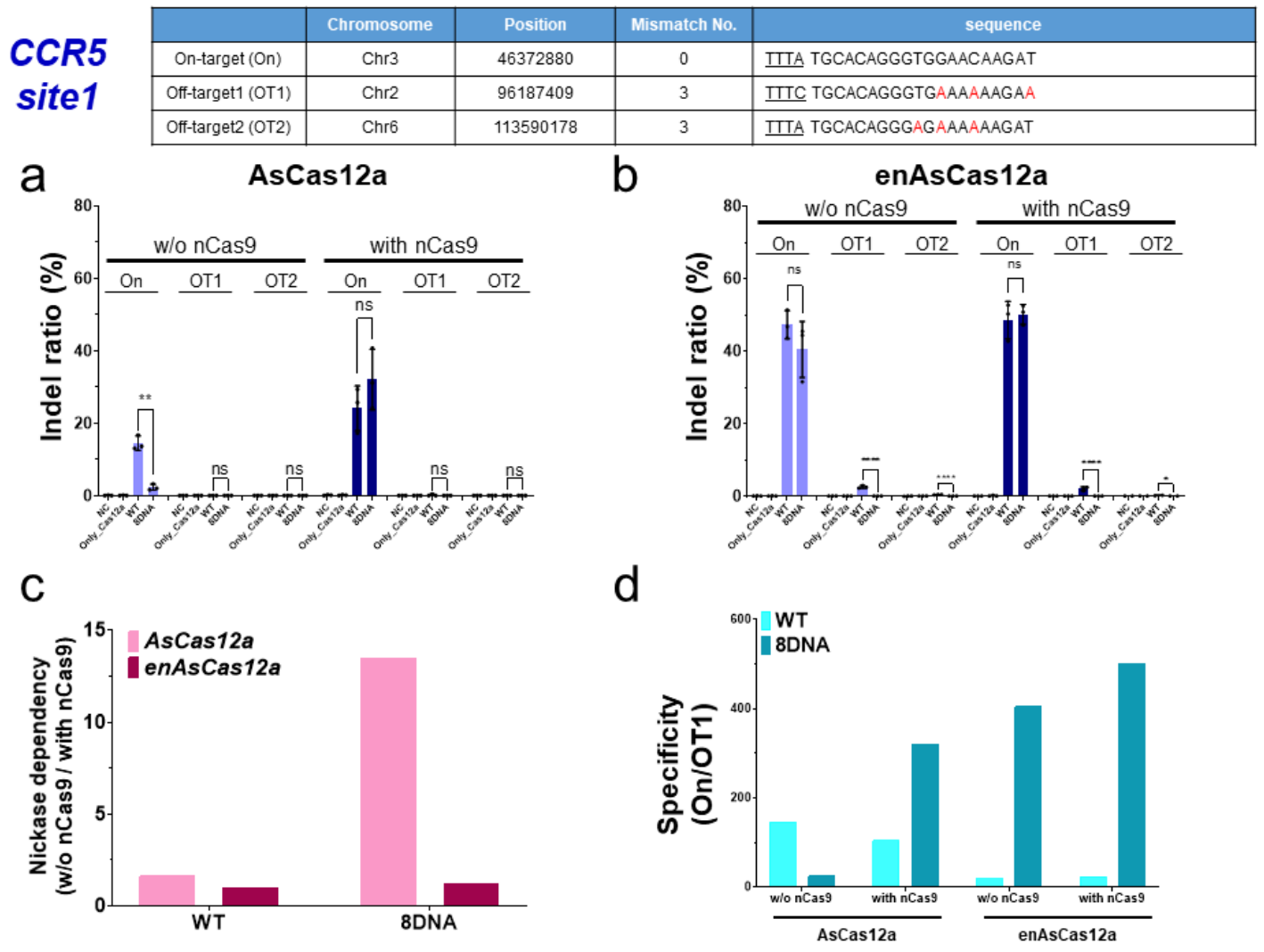

\begin{tabular}{|c|c|c|c|c|c|c|}
\hline \multirow{4}{*}{ AAVS1 } & & Chromosome & Position & Bulge & MismatchNo. & Sequence \\
\hline & On-target(On) & Chr19 & 55115578 & 0 & 0 & IIIG CTTACGATGGAGCCAGAGAG \\
\hline & Off-target1 (OT1) & Chr4 & 95395972 & 0 & 3 & IIIC CTIATGATGAAGCCAGAGAA \\
\hline & Off-target1 (OT2) & Chr1 & 182157128 & RNA - 1 & 2 & IIIA CTA-GATGAAGCCACAGAG \\
\hline
\end{tabular}

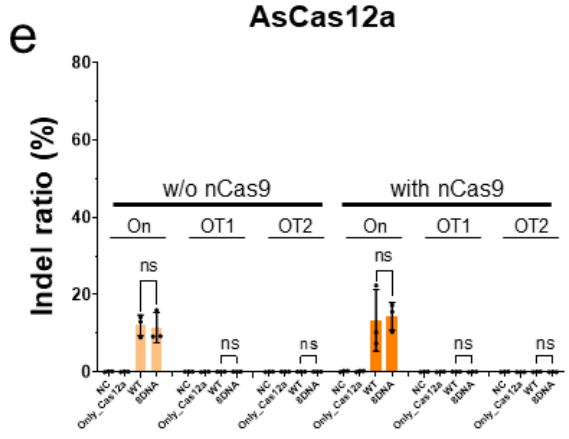

$f$

enAsCas12a

g

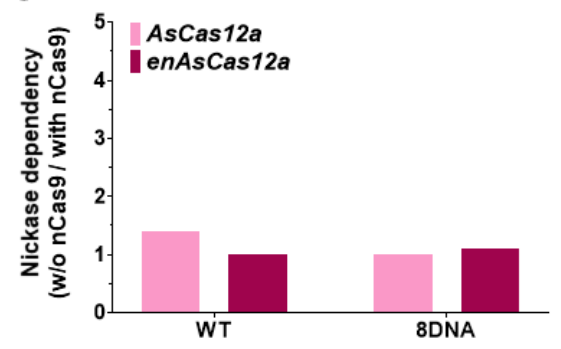

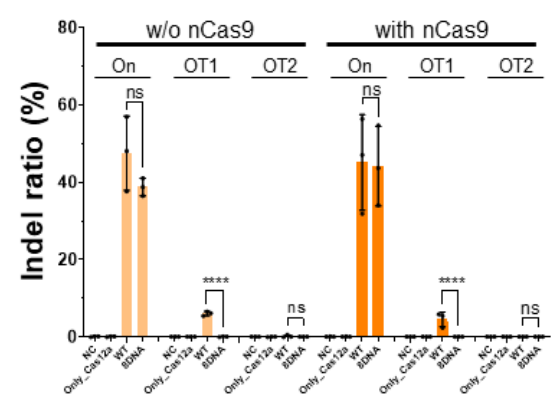

$\mathrm{h}$

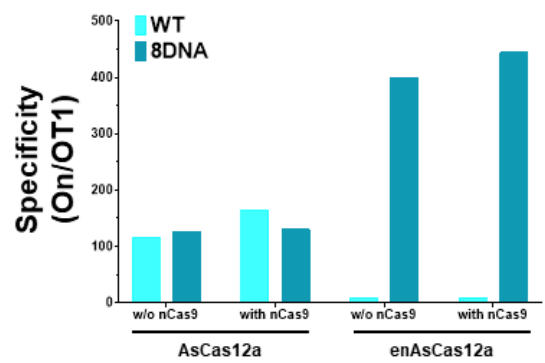




\begin{tabular}{|c|c|c|c|c|c|c|}
\hline \multirow{5}{*}{$\begin{array}{c}\text { DNMT1 } \\
\text { site2 }\end{array}$} & & Chromosome & Position & Bulge & Mismatch No. & Sequence \\
\hline & On-target (On) & Chr19 & 10133920 & 0 & 0 & IITG GCTCAGCAGGCACCTGCCTC \\
\hline & Off-target1 (OT1) & Chr21 & 25149419 & 0 & 2 & CTA GCTCAGCAGGCACCTGCCCA \\
\hline & Off-target2 (OT2) & Chr12 & 57638266 & 0 & 4 & IIIA GCTCAGCTGACACCTGCCCA \\
\hline & Off-target3 (ОТ3) & Chr6 & 107959600 & 0 & 2 & ICCA GCTCAGCAGACACCAGCCTC \\
\hline
\end{tabular}

i
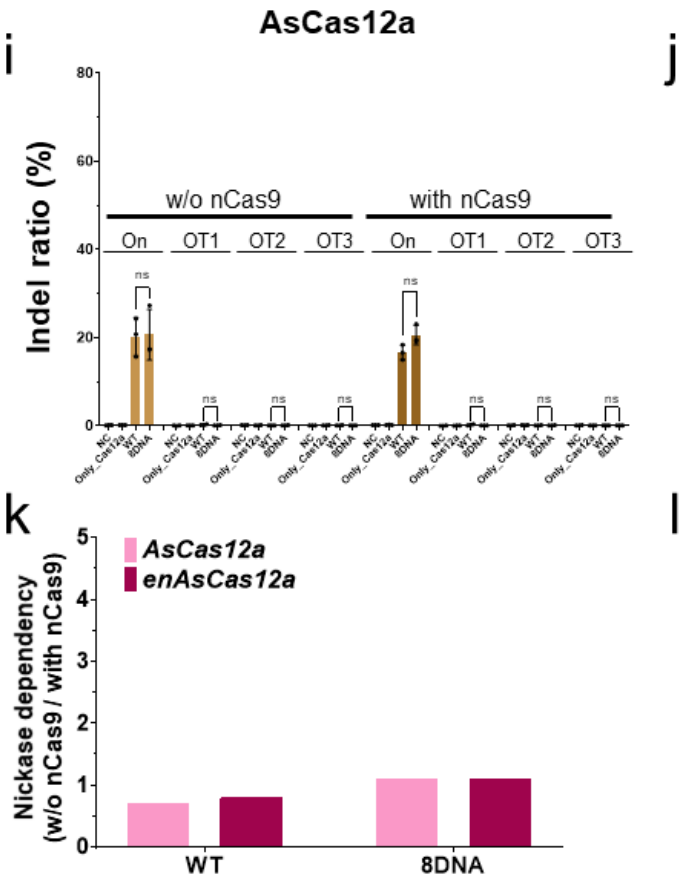

enAsCas12a
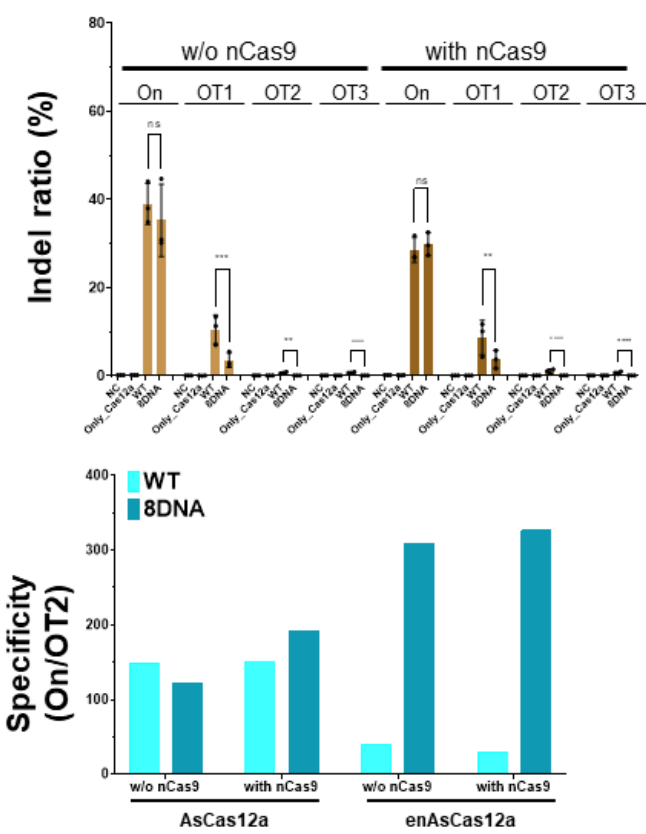

Figure 3. Comparison of genome editing specificity of en-AsCas12a and wt-AsCas12a based on chimeric DNA-RNA guide targeting intracellular genome. a-l, Comparison of genome editing target specificity (on-target editing(\%) / off-target editing(\%)) of wt-AsCas12a and en-AsCas12a on genome in human cell line(HEK293FT) using an optimized chimeric DNA-RNA guide(8DNA). a-d, Comparison of target specificity between wt- and en-AsCas12a on the intracellular genome target sequence (CCR5-site1) using wt-crRNA(WT) and 3'-end 8nt DNA substituted crRNA(8DNA). e-h, Comparison of target specificity on the target sequence (AAVS1-site1). i-l, Comparison of target specificity on the target sequence (DNMT1-site2). Data are shown as means \pm s.e.m. from three independent experiments. $P$-values are calculated using a two-way ANOVA with sidak's multiple comparisons test (ns: not significant, $P^{*}:<0.0332, P^{* *}:<0.0021, P^{* * *}:<0.0002, P^{* * * *}:<0.0001$ ). NC: negative control, only Cas12a: only protein treated, WT: Wild-type crRNA was treated with wt- or enAsCas12a, 8DNA: Chimeric crRNA (sequential 8DNA substitution at 3'-end of crRNA) was treated with wt- or en-AsCas12a. nCas9: nickase Cas9(D10A), Nickase dependency = (w/o nCas9 editing(\%) / w/ nCas9 editing(\%)), Specificity $=$ (on-target editing(\%) / off-target editing(\%)). 


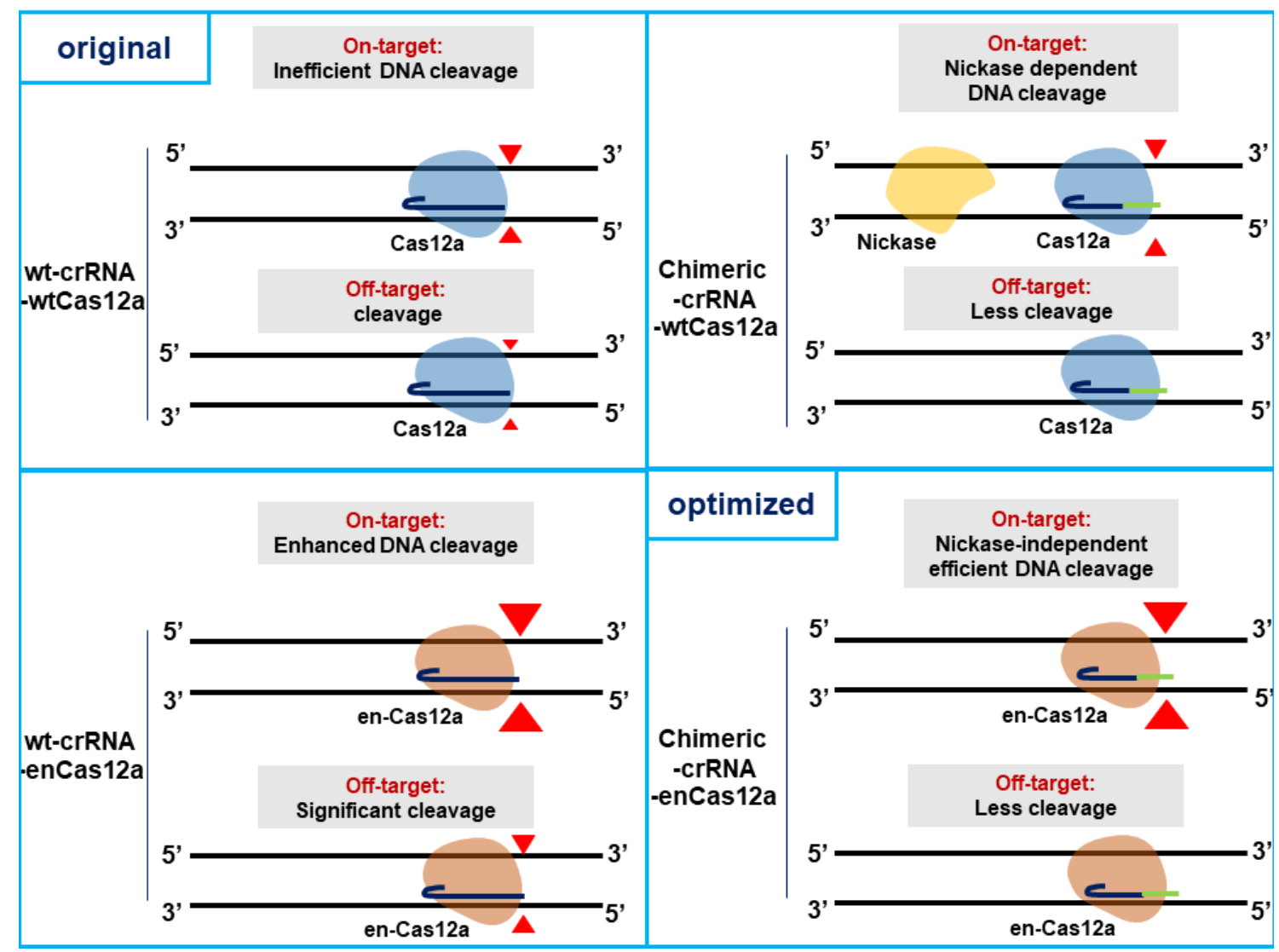

Figure 4. A model for enhancing target specificity and editing efficiency of en-Cas12a based on chimeric DNA-RNA guided engineering. wt-crRNA guided wt-Cas12a system: In general, the wtCas12a effector can induce genetic mutations in target sequences, but can also induce mutations in similar off-target sequences., wt-crRNA guided en-Cas12a system: Recognition of a target sequence is enhanced by a effector engineered by amino acid substitution, and thus the efficiency of inducing gene mutations is increased compared to that of the wt-Cas12a effector. However, due to the same principle as target sequence recognition, there is a problem in that the mutation induction efficiency of off-target sequences is also increased., chimeric-crRNA guided wt-Cas12a system: Effectively reduced off-target mutation induction efficiency when using a chimeric DNA-RNA guide with 8nt DNA substituted at the 3 -end. However, the efficiency of mutation induction for the target nucleotide sequence in the genome is also reduced, so the efficiency is restored only when there is the action of nickase on the nucleotide sequence near the target., chimeric-crRNA guided en-Cas12a system: Maximizes the target sequence indel ratio(\%) and minimizes the off-target indel ratio(\%) when using a chimeric DNA-RNA(8DNA) guided en-Cas12a effector which is engineered by amino acid substitution. It can induce more accurate and high-efficiency gene editing than wt-Cas12a on genomic DNA. DNA cleavage points are indicated by red arrows, and the degree of cleavage is indicated by arrow size according to the Cas12a activity. The wt-Cas12a and en-Cas12a effectors are shown in blue and brown, respectively. In the wt-crRNA and chimeric DNA-RNA guides, RNA is indicated in dark blue, and nucleotides replaced with DNA are indicated in green. 
a

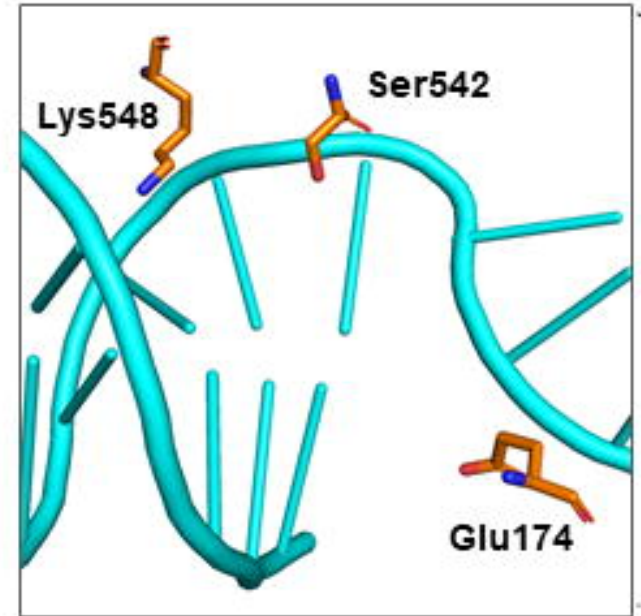

Residues in PI near site

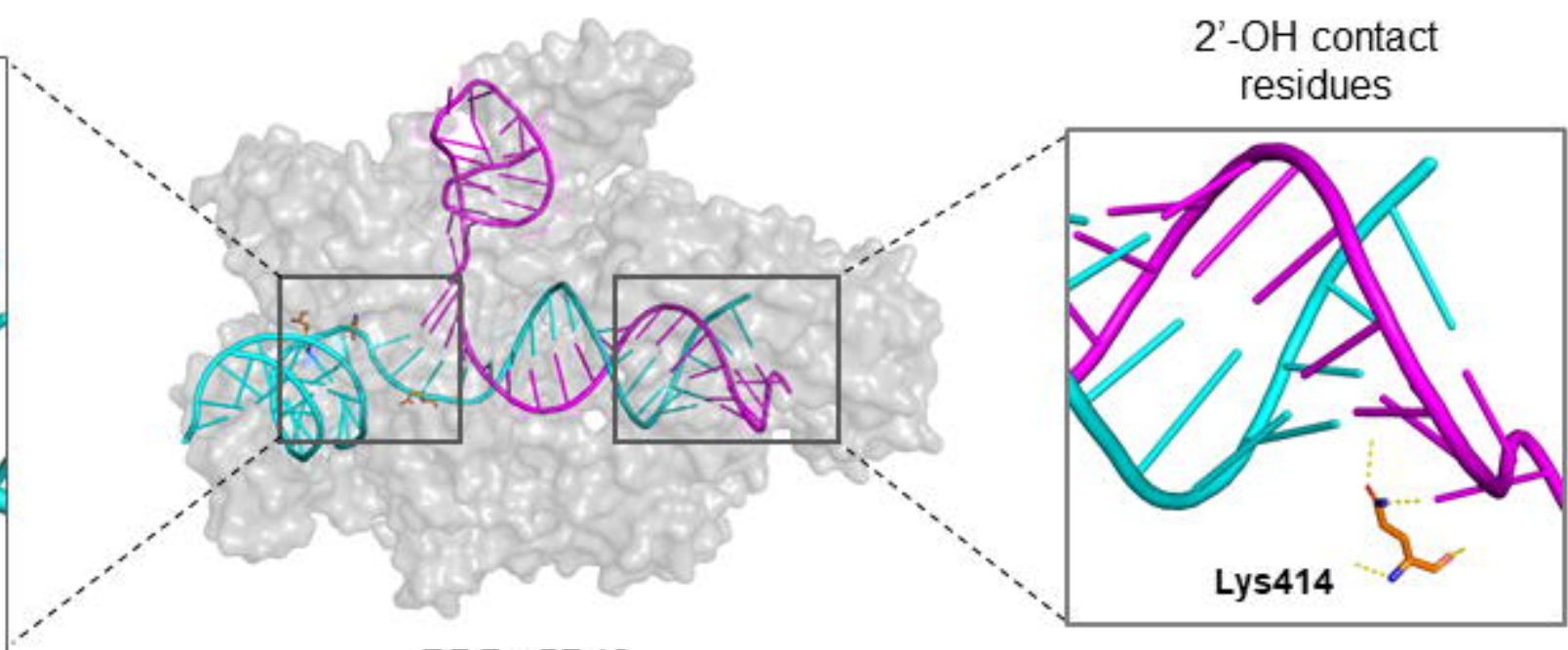

PDB : 5B43

b
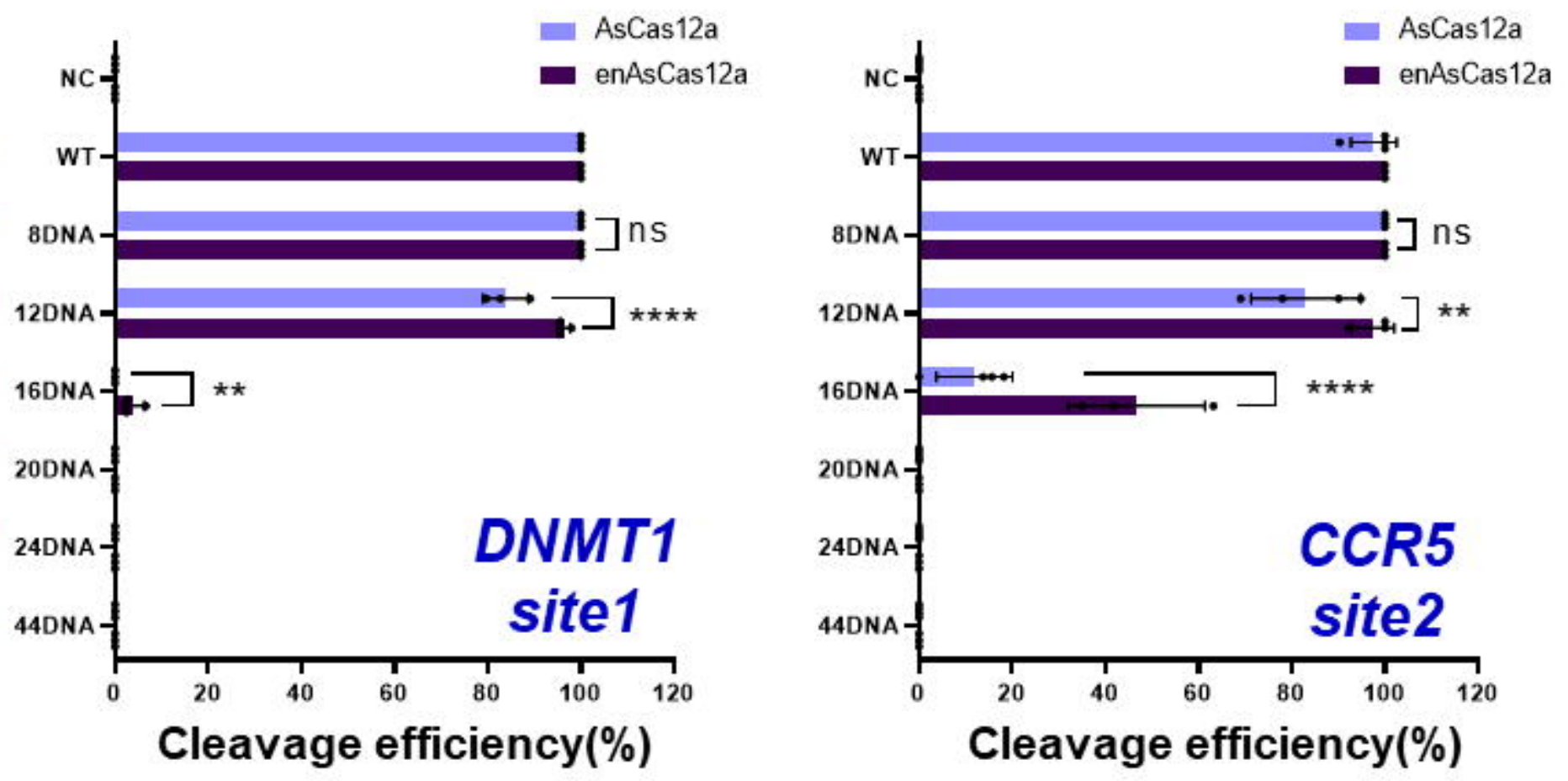
a
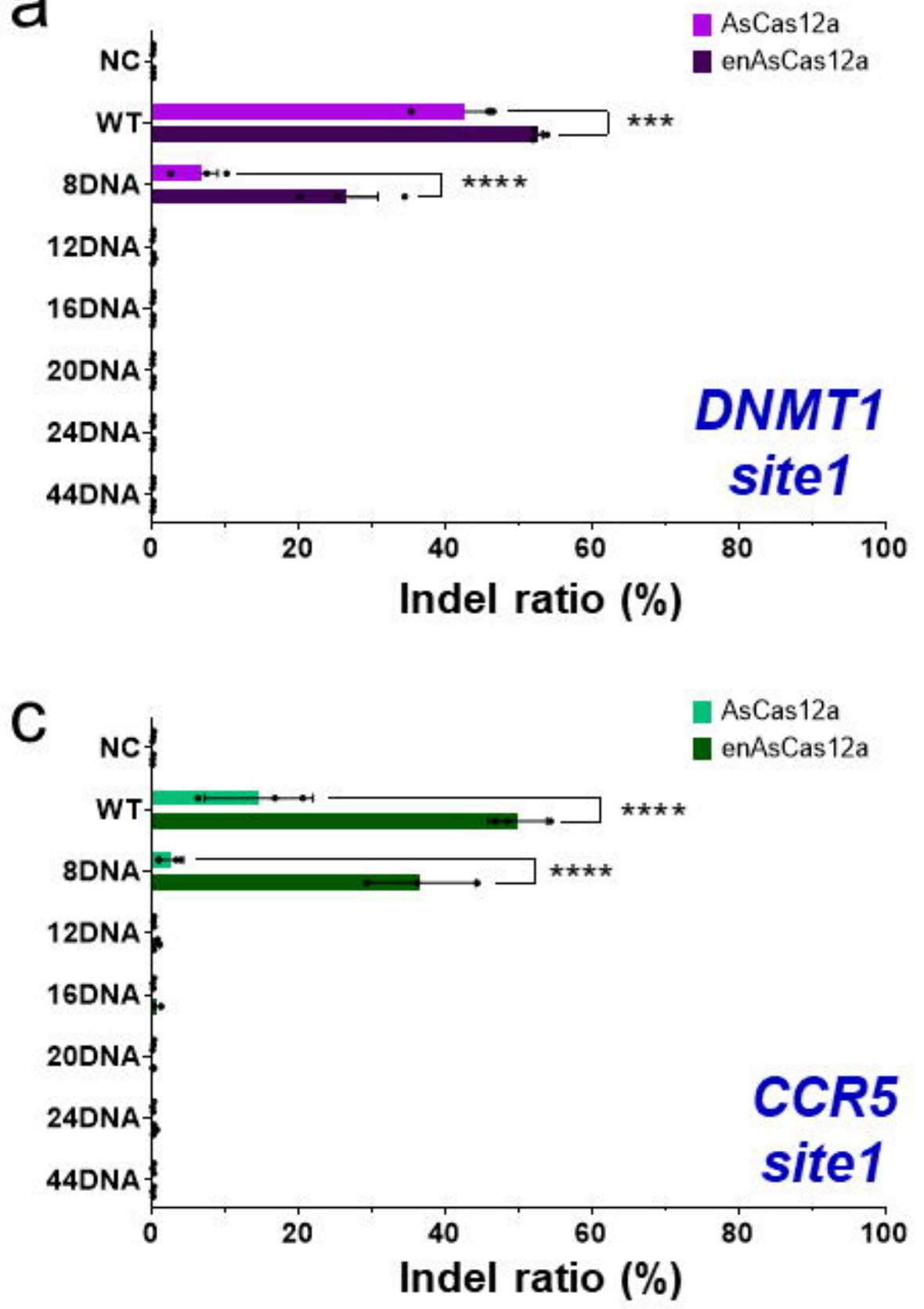

b

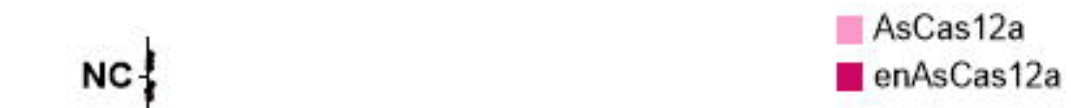

IL12A-AS1 44DNA-

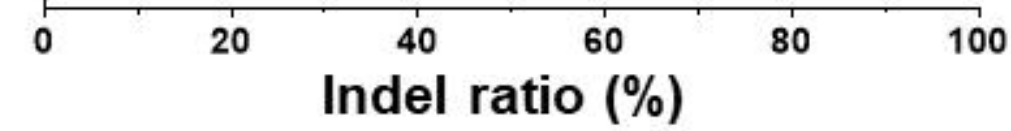




\begin{tabular}{|c|c|c|c|c|l|}
\cline { 2 - 6 } CCR5 & & Chromosome & Position & Mismatch No. & \\
\cline { 2 - 6 } \multirow{3}{*}{ site1 } & On-target (On) & Chr3 & 46372880 & 0 & ITTA TGCACAGGGTGGAACAAGAT \\
\cline { 2 - 6 } & Off-target1 (OT1) & Chr2 & 96187409 & 3 & ITTC TGCACAGGGTGAAAAAAGAA \\
\cline { 2 - 6 } & Off-target2 (OT2) & Chr6 & 113590178 & 3 & ITTA TGCACAGGGAGAAAAAAGAT \\
\cline { 2 - 6 } & &
\end{tabular}

a

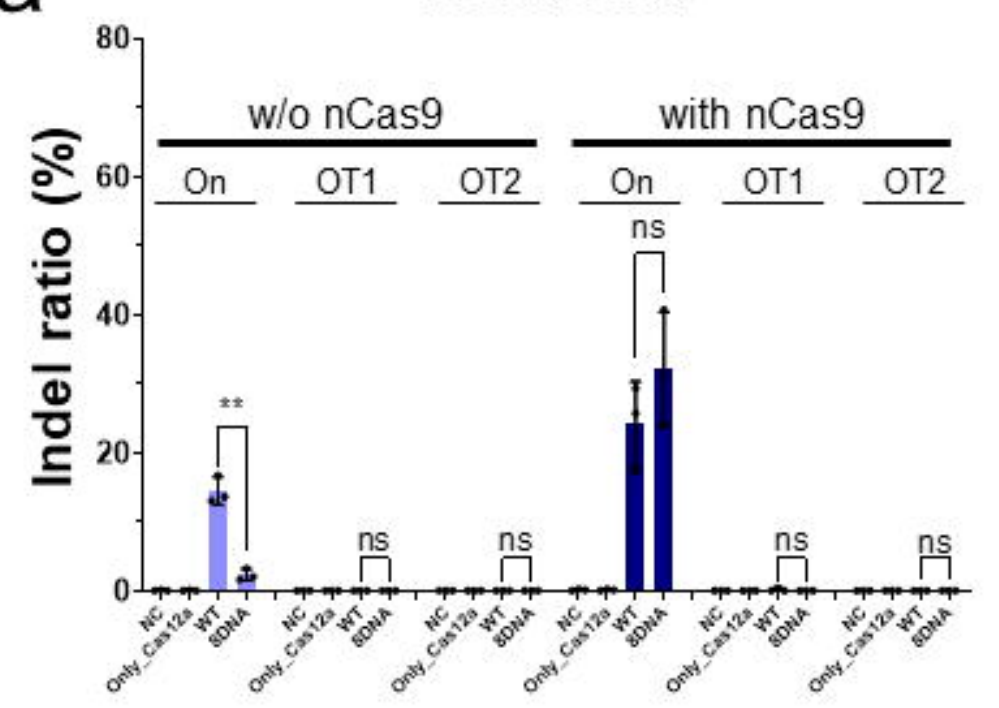

C

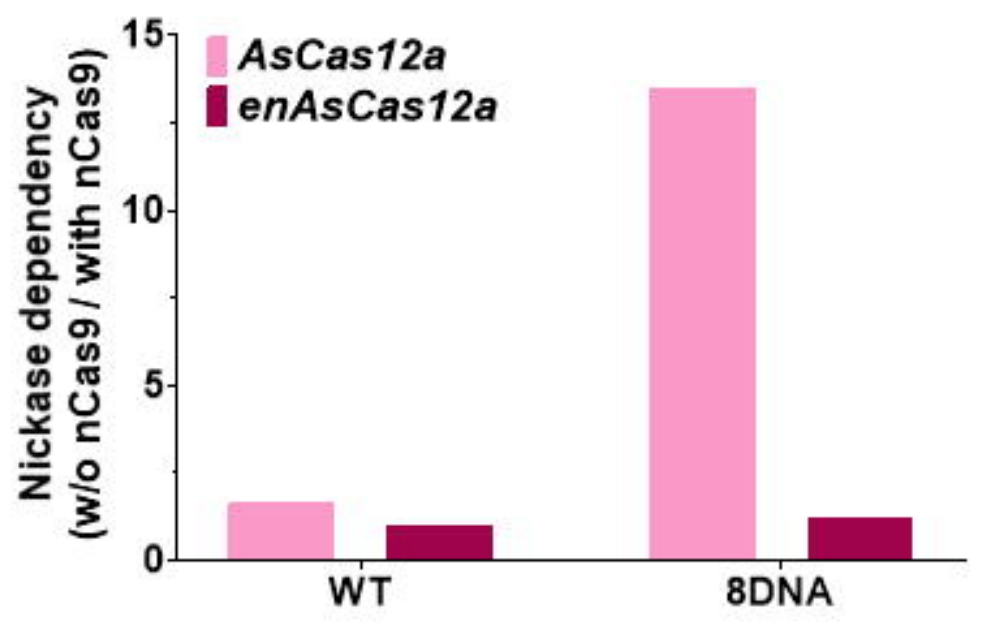

b

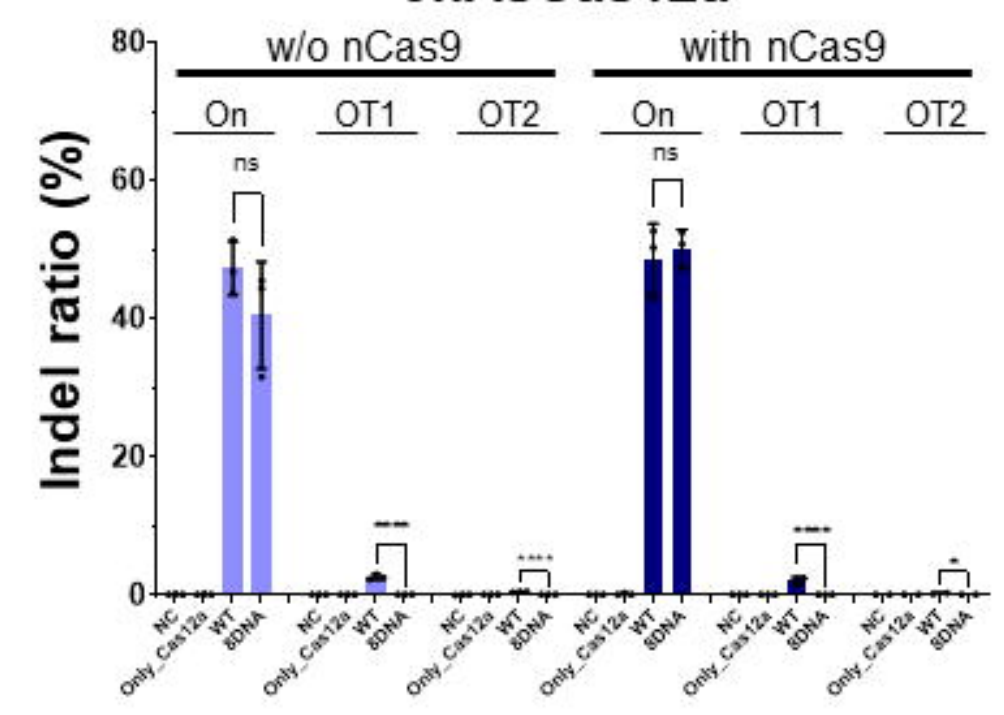

d

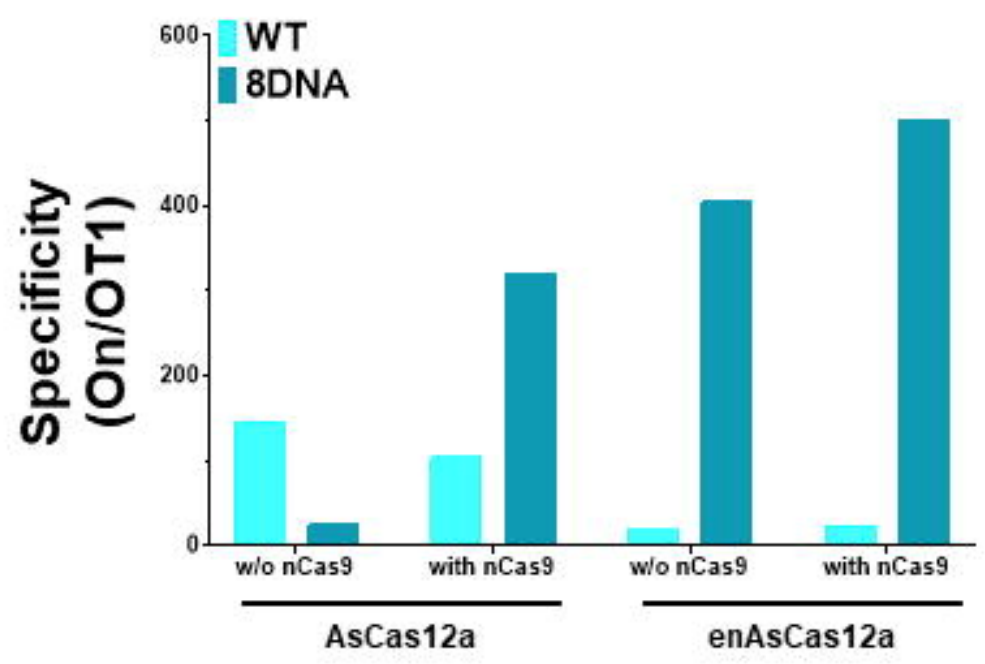




\begin{tabular}{|c|c|c|c|c|c|c|}
\hline \multirow{4}{*}{ AAVS1 } & & Chromosome & Position & Bulge & MismatchNo. & Sequence \\
\hline & On-target $(0 n)$ & Chr19 & 55115578 & 0 & 0 & IITG CTTACGATGGAGCCAGAGAG \\
\hline & Off-target1 (OT1) & $\mathrm{Chr} 4$ & 95395972 & 0 & 3 & IITC CПTATGATGAAGCCAGAGAA \\
\hline & Off-target1 (OT2) & Chr1 & 182157128 & RNA - 1 & 2 & IITA CTIA-GATGAAGCCACAGAG \\
\hline
\end{tabular}

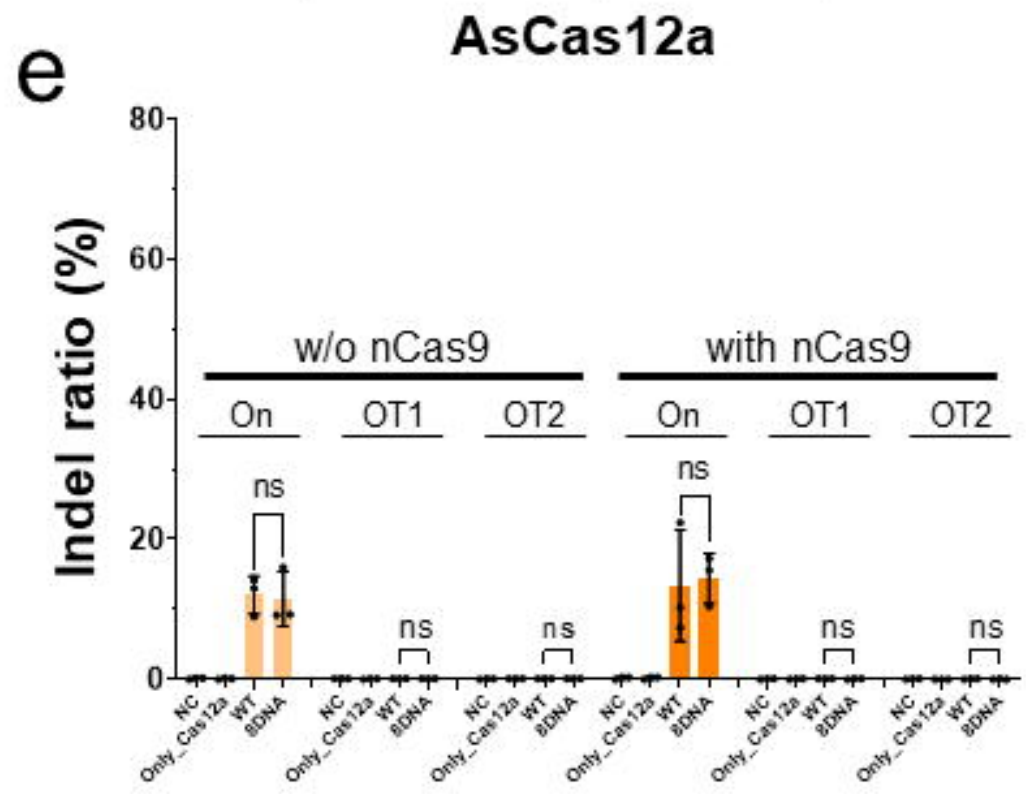

f

\section{enAsCas12a}

g

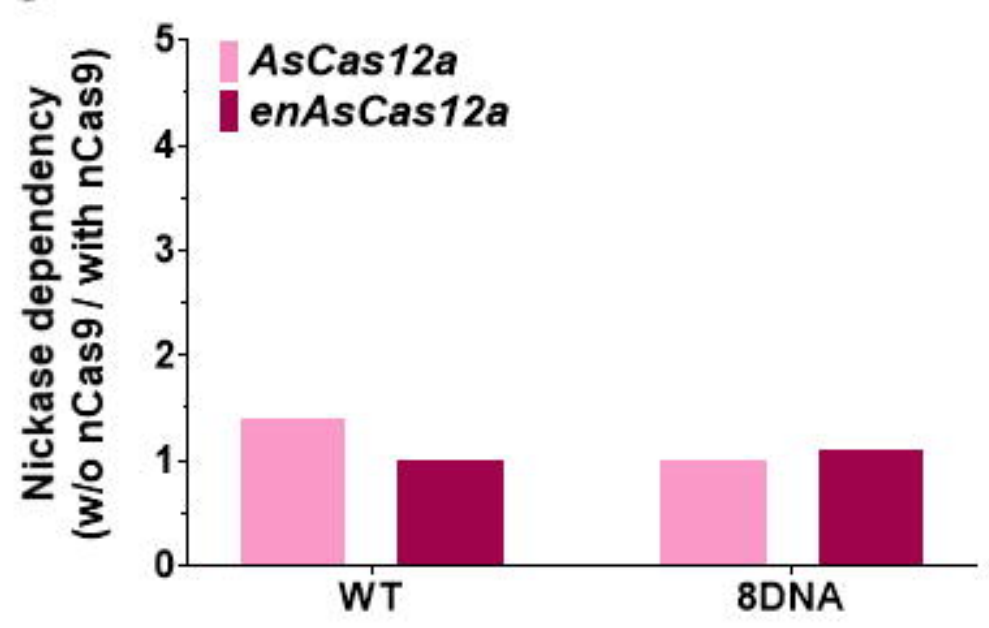

h
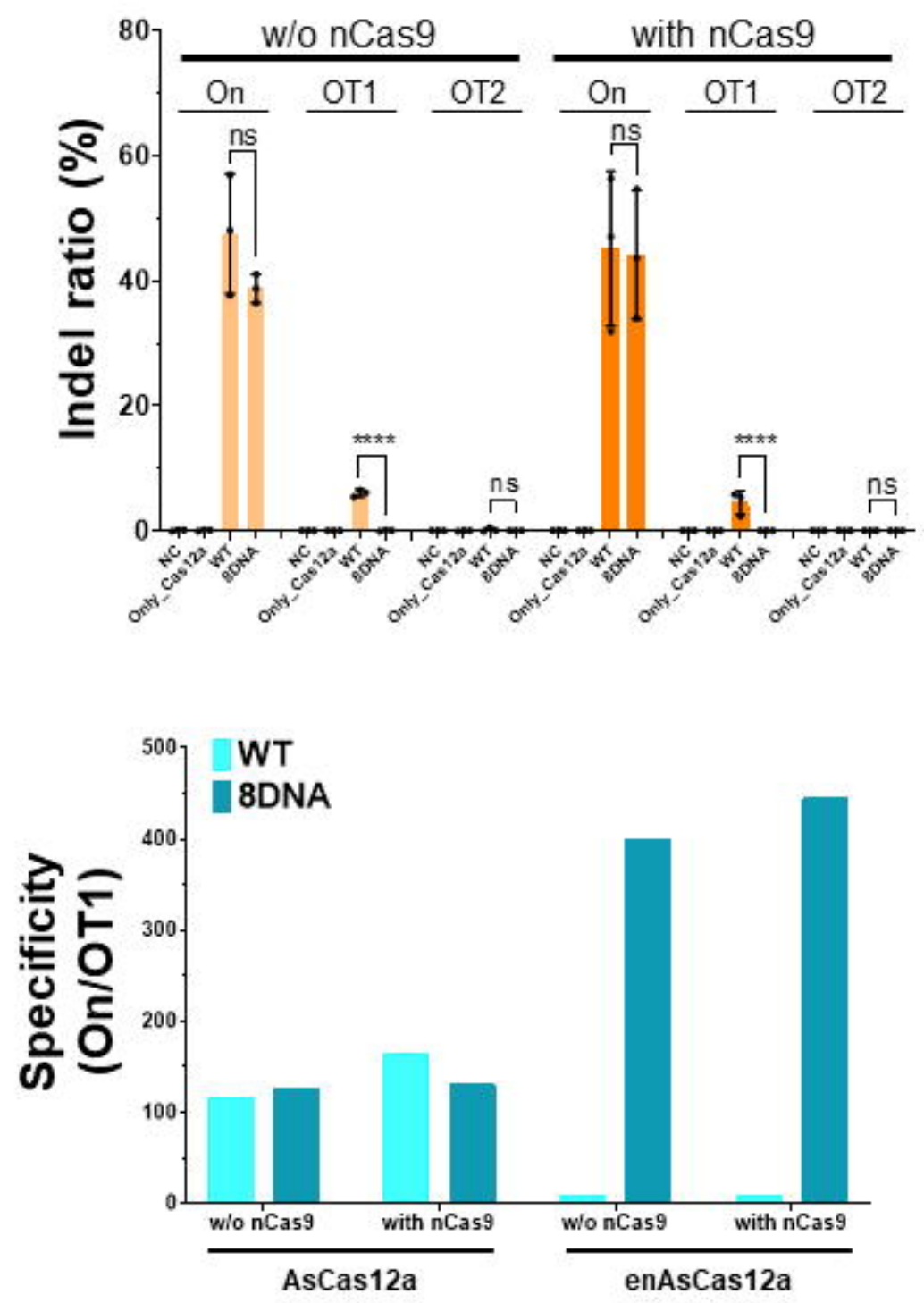


\begin{tabular}{|c|c|c|c|c|c|c|}
\hline \multirow{5}{*}{$\begin{array}{c}\text { DNMT1 } \\
\text { site2 }\end{array}$} & & Chromosome & Position & Bulge & MismatchNo. & Sequence \\
\hline & On-target $(O n)$ & Chr19 & 10133920 & 0 & 0 & IITG GCTCAGCAGGCACCTGCCTC \\
\hline & Off-target1 (OT1) & Chr21 & 25149419 & 0 & 2 & CTIA GCTCAGCAGGCACCTGCCCA \\
\hline & Off-target2 (OT2) & Chr12 & 57638266 & 0 & 4 & IIIA GCTCAGCTGACACCTGCCCA \\
\hline & Off-target 3 (OT3) & Chr6 & 107959600 & 0 & 2 & TCCA GCTCAGCAGACACCAGCCTC \\
\hline
\end{tabular}

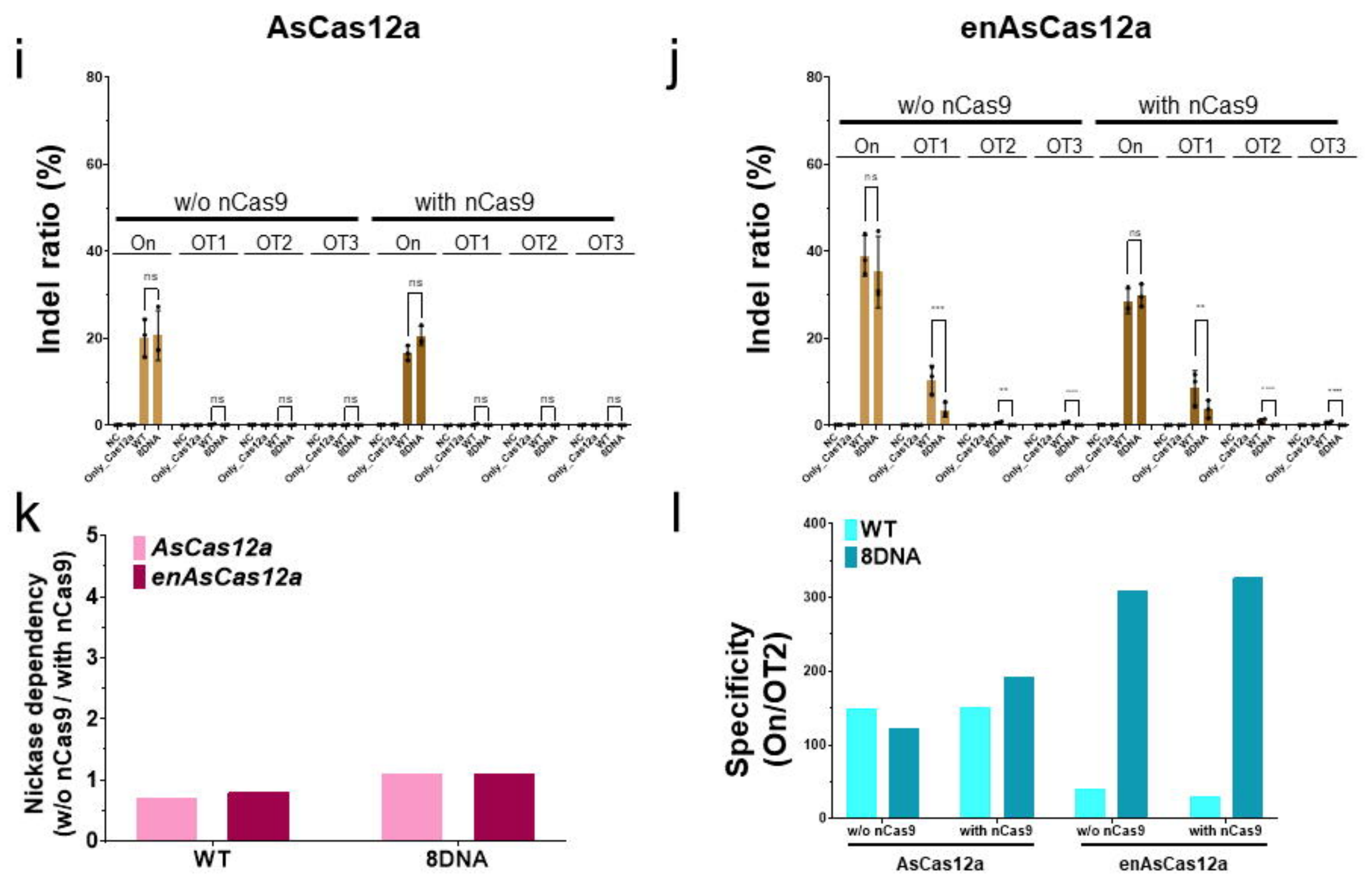


On-target:

Inefficient DNA cleavage

wt-crRNA -wtCas12a

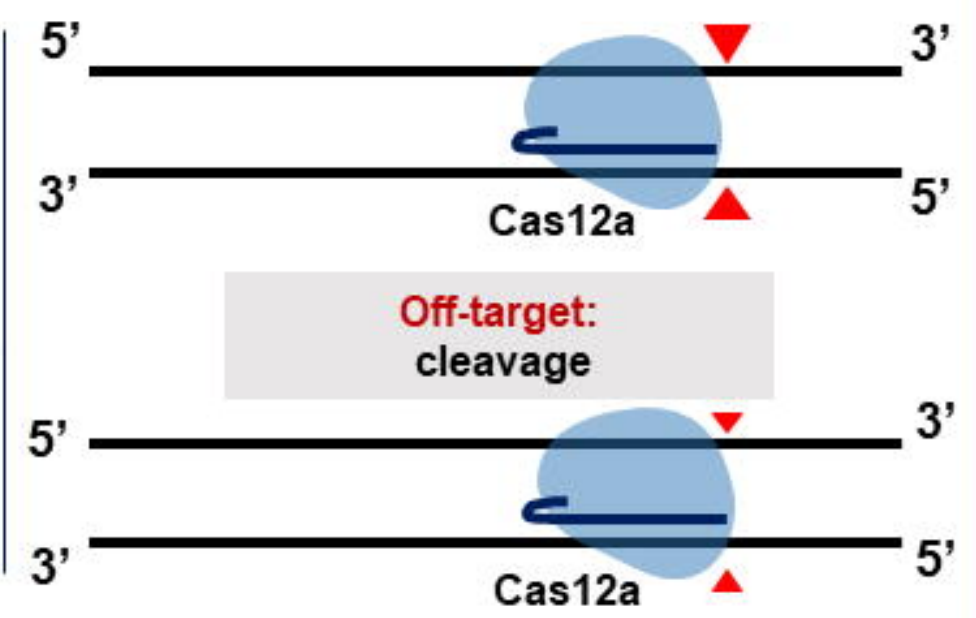

On-target:

Enhanced DNA cleavage

wt-crRNA -enCas12a

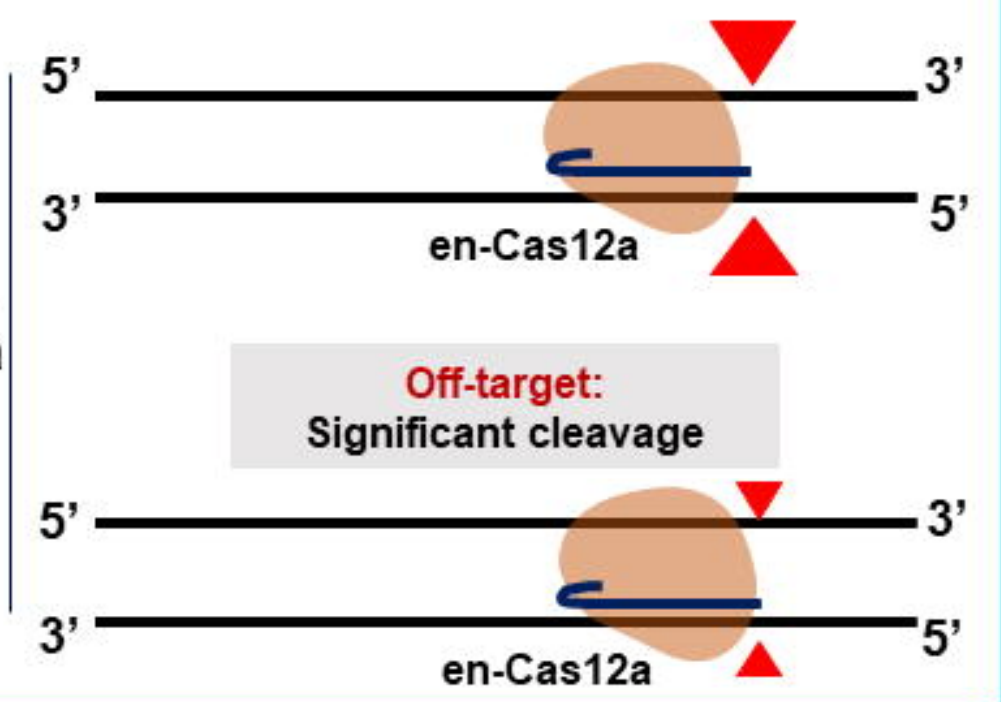

On-target:

Nickase dependent

DNA cleavage

Chimeric

-crRNA

-wtCas12a

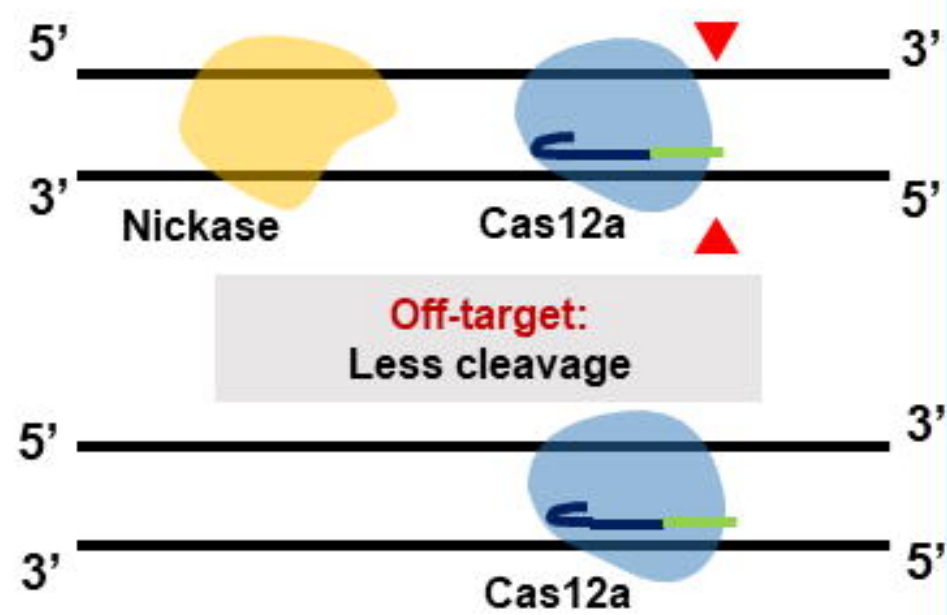

optimized

Chimeric

-crRNA

-enCas12a

5

On-target:

Nickase-independent efficient DNA cleavage

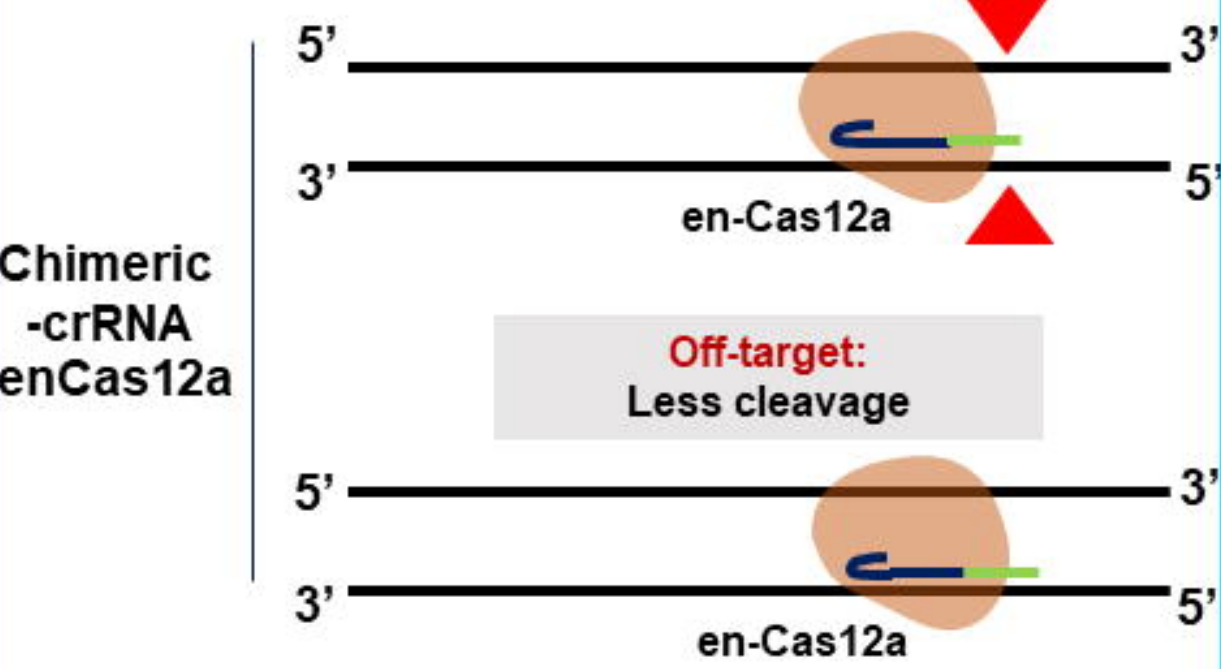

